\title{
What is known about paediatric nurse burnout: a scoping review
}

\author{
Laura Buckley $^{1,2^{*}}$ (D), Whitney Berta ${ }^{3}$, Kristin Cleverley ${ }^{1,4}$, Christina Medeiros ${ }^{1}$ and Kimberley Widger ${ }^{1,2}$
}

\begin{abstract}
Burnout in healthcare providers has impacts at the level of the individual provider, patient, and organization. While there is a substantial body of literature on burnout in healthcare providers, burnout in pediatric nurses has received less attention. This subpopulation may be unique from adult care nurses because of the specialized nature of providing care to children who are typically seen as a vulnerable population, the high potential for empathetic engagement, and the inherent complexities in the relationships with families. Thus, the aim of this scoping review was to investigate, among pediatric nurses, (i) the prevalence and/or degree of burnout, (ii) the factors related to burnout, (iii) the outcomes of burnout, and (iv) the interventions that have been applied to prevent and/or mitigate burnout. This scoping review was performed according to the PRISMA Guidelines Scoping Review Extension. CINAHL, EMBASE, MEDLINE, PsycINFO, ASSIA, and The Cochrane Library were searched on 3 November 2018 to identify relevant quantitative, qualitative, and mixed-method studies on pediatric nurse burnout. Our search identified 78 studies for inclusion in the analysis. Across the included studies, burnout was prevalent in pediatric nurses. A number of factors were identified as impacting burnout including nurse demographics, work environment, and work attitudes. Similarly, a number of outcomes of burnout were identified including nurse retention, nurse well-being, patient safety, and patient-family satisfaction. Unfortunately, there was little evidence of effective interventions to address pediatric nurse burnout. Given the prevalence and impact of burnout on a variety of important outcomes, it is imperative that nursing schools, nursing management, healthcare organizations, and nursing professional associations work to develop and test the interventions to address key attitudinal and environmental factors that are most relevant to pediatric nurses.
\end{abstract}

Keywords: Burnout, Burn out, Work stress, Pediatric nurses, Pediatrics, Pediatrics, Nurses

Burnout has been a widely studied topic of interest over the last 40 years, with significant resources devoted toward investigating its causes, impacts, and strategies for mitigation [1]. Burnout is a work outcome, defined by prolonged occupational stress in an individual that presents as emotional exhaustion, depersonalization, and diminished personal accomplishment [2].

The study of burnout in healthcare professionals is important as it has impacts at the level of the individual provider [3-5], the patient [6-9], and the organization $[5,10-12]$. As nurses make up the largest group of healthcare professionals, there have been a number of

\footnotetext{
* Correspondence: laura.buckley@sickkids.ca

'Lawrence S. Bloomberg Faculty of Nursing, University of Toronto, 155

College Street, Toronto, ON M5T 3M6, Canada

${ }^{2}$ Hospital for Sick Children, 555 University Avenue, Toronto, ON M5G 1X8,

Canada

Full list of author information is available at the end of the article
}

studies that have explored contributing factors [13] and interventions for their burnout [14]. Pediatric nurses are a lesser-studied population, perhaps due to the relatively small number of pediatric nurses compared to general service nurses and the broader population of healthcare professionals. Burnout in pediatric nurses may be unique from adult care nurses because of the specialized nature of providing care to children who are typically seen as a vulnerable population, the high potential for empathetic engagement, and the inherent complexities in the relationships with families $[15,16]$. Only one literature review could be located on the topic of pediatric nurse burnout; it mainly focused on burnout prevalence, which was found to be moderate to high [17]. Further synthesis of the literature in other domains of the topic is needed to explore factors associated with pediatric nurse burnout, the associated outcomes, and interventions.

(C) The Author(s). 2020 Open Access This article is distributed under the terms of the Creative Commons Attribution 4.0 International License (http://creativecommons.org/licenses/by/4.0/), which permits unrestricted use, distribution, and 
The purpose of this scoping review is to explore what is known about pediatric nurse burnout to guide future research on this highly specialized population and, ultimately, improve both pediatric nurse and patient wellbeing. More specifically, the aim of this scoping review was to investigate, among pediatric nurses, (i) the prevalence and/or degree of burnout, (ii) the factors related to burnout, (iii) the outcomes of burnout, and (iv) the interventions that have been applied to prevent and/or mitigate burnout.

\section{Methods}

\section{Protocol registration}

This scoping review was performed according to the Preferred Reporting Items for Systematic Reviews and Meta-Analyses (PRISMA) guidelines scoping review extension [18]. The protocol was registered on Open Science Framework on 25 March 2019 and can be accessed at https://osf.io/5xrkg/.

\section{Information sources and search strategy}

In consultation with an experienced librarian, the following electronic databases were searched on 3 November 2018 without limitation to a publication date range in order to maximize inclusion: The Cochrane Library, CINAHL, EMBASE, MEDLINE, PsycINFO, and ASSIA. All electronic database search strategies used in this review can be found in Appendix A. The term "pediatrics" was not part of the electronic database search to avoid inadvertently excluding studies that contained pediatric nurses as a non-primary subject group. The selected articles from the electronic database search were screened for inclusion of the pediatric nurse population. For the purposes of this review, the pediatric patient population is defined as newborn to age 21 as defined by the American Academy of Pediatrics, acknowledging that this age range may be slightly extended based on the country and patient needs [19].

\section{Eligibility criteria}

All qualitative, quantitative, or mixed-methods studies published in English that examined the prevalence and/ or degree of burnout in pediatric nurses using selfidentification or self-report assessment tools were included. Commentaries, letters, and editorials were excluded as these are not peer-reviewed and often referred to colloquial definitions, not the clinical definition of burnout of interest in this scoping review. Dissertations were excluded, but their corresponding publications were screened for inclusion. Conference abstracts were excluded as they are often inconsistent with their corresponding full reports [20]. Systematic or scoping reviews and meta-synthesis were excluded, but references were hand-screened for suitable studies.

\section{Selection of sources of evidence}

All citations retrieved from the databases were uploaded into Endnote with duplicates removed as per protocol [21]. The remaining citations were uploaded into Covidence for review by the research team (LB, CM, KW). Titles and abstracts were independently reviewed against the selection criteria in a blinded process by two reviewers (LB and $\mathrm{CM})$. The remaining citations were then reviewed as full-text articles for inclusion against the selection criteria in a blinded process by two reviewers (LB and $\mathrm{CM}$ ). Disagreements were resolved by a third reviewer $(\mathrm{KW})$.

\section{Data charting process}

Data were extracted from included articles and entered into a Microsoft Excel spreadsheet. Extraction was performed by one researcher (LB). The following data items were extracted: title, journal, authors, year of publication, country of publication, sample size, study aim, study design, tool used to measure burnout, burnout prevalence and scores, factors that contribute to the development of burnout in pediatric nurses, factors that prevent or mitigate burnout in pediatric nurses, the impact of burnout in pediatric nurses, and interventions for pediatric nurse burnout.

\section{Synthesis of results}

A quantitative synthesis specific to the prevalence and degree of burnout was completed based on the included articles that reported raw scores for any of the Maslach Burnout Inventory (MBI) subscales. A mean score was calculated by hand across studies for each subscale, by totaling the raw scores and dividing by the total number of studies that included a raw score for that subscale. The resulting mean was also categorized as low, moderate, or high burnout based on published cutoff scores [22]. Other data were synthesized qualitatively to map current evidence available to address the remaining study aims. Aims ii and iii were analyzed using directed content analysis [23] following the themes outlined by Berta et al. [24], work environment, work attitudes, and work outcomes. Aim iv data was synthesized by grouping together similar interventions and descriptively summarizing the interventions that were effective in reducing burnout. Given that the overall purpose of the review was to explore the breadth of what is currently known about burnout in pediatric nurses, a quality assessment of individual studies was not conducted [18].

\section{Results}

Description of the search and demographics of studies included

Through the initial database search, 16909 possible papers were identified. After deduplication, 8629 titles/ 
abstracts were screened and 1206 articles were assessed for eligibility at the level of full-text screening. After applying the inclusion and exclusion criteria, a total of 78 studies [16, 25-101] were deemed relevant and retained for analysis (Fig. 1). The characteristics of included studies are provided in Table 1. Publication dates ranged from 1981 to 2018, with the majority published between 2009 and 2017. The number of pediatric nurses who participated as either a primary or sub-sample ranged from five to 3710 . The most common study design was cross-sectional $(n=60)$, with 10 studies using multi- or mixed methods, seven using an interventional design, and one each using case-control, exploratory prospective, and longitudinal designs. Only 45 of the 78 studies reviewed used exclusive samples of pediatric nurses; the remaining studies only included pediatric nurses as a subpopulation of a larger sample. The results in this review are reported for pediatric nurse samples and subsamples only. Almost half (46\%) of the included studies were conducted in the USA, followed by Canada $(n=7)$, China $(n=5)$, Turkey $(n=3)$, Brazil $(n=3)$, Taiwan $(n=$ $2)$, Australia $(n=2)$, and Switzerland $(n=2)$, plus 18 other countries where only a study was conducted. Out of the 78 studies included, 53 (68\%) used some form (either complete or abbreviated) of the MBI to measure burnout (see Table 1).

\section{Burnout prevalence and scores}

Although all of the included studies measured burnout using a self-report assessment tool or binary selfidentification, only 65 reported burnout scores for a sample of pediatric nurses (Table 2). In total, 53 studies used the MBI, 34 reported on the Emotional Exhaustion subscale with 24 reporting raw scores [25, 27, 29, 39, 42,

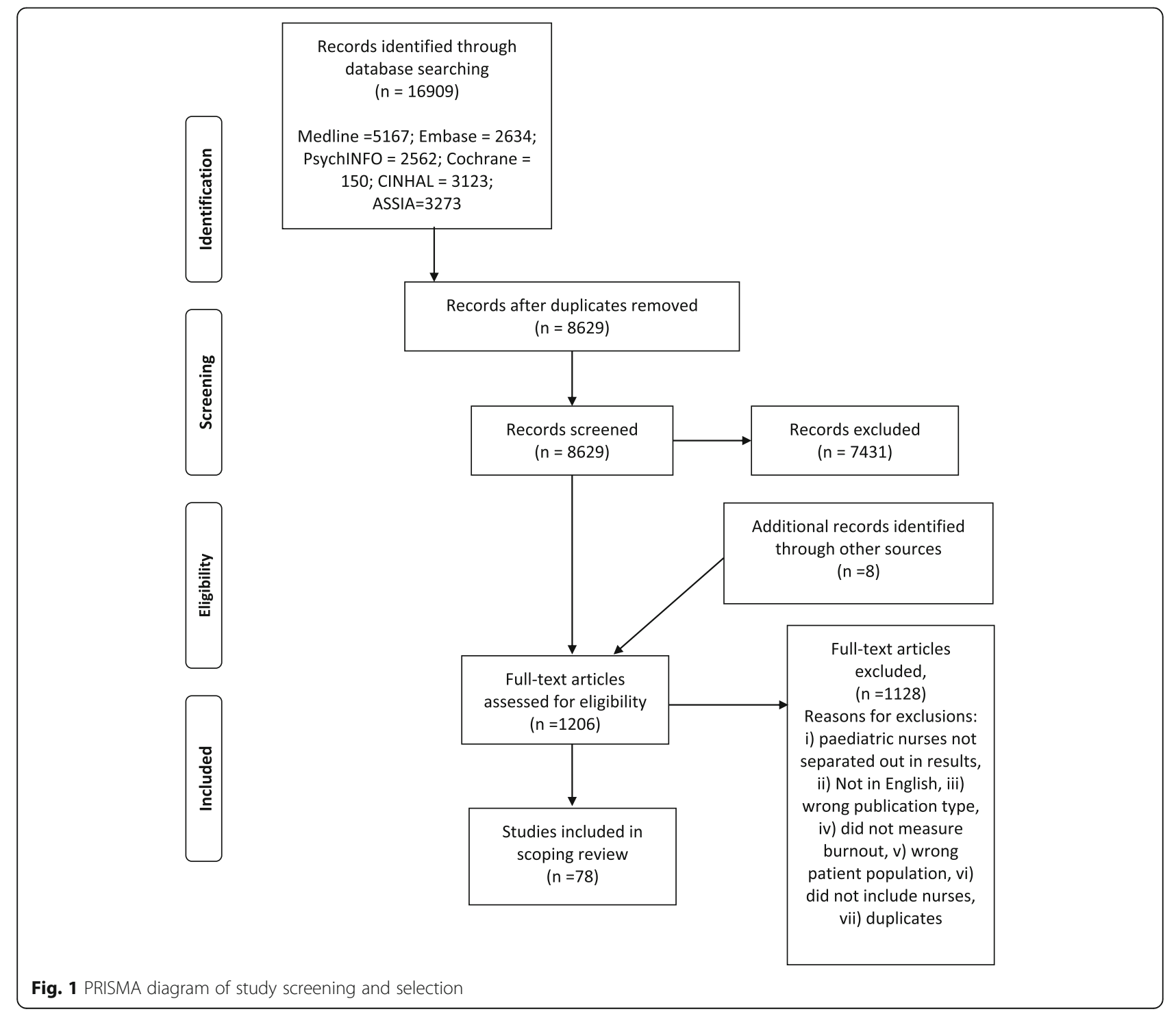


Table 1 Characteristics of 78 studies exploring pediatric nurse burnout included in the scoping review

\begin{tabular}{|c|c|c|c|}
\hline $\begin{array}{l}\text { Author(s), year, } \\
\text { country }\end{array}$ & Sample & Study design & $\begin{array}{l}\text { Burnout measurement } \\
\text { tool }\end{array}$ \\
\hline $\begin{array}{l}\text { Adwan, } 2014[25] \\
\text { (USA) }\end{array}$ & $\begin{array}{l}120 \text { nurses from a large academic medical center, from four } \\
\text { pediatric patient care units and pediatric float pool nurses }\end{array}$ & Cross-sectional correlational design & MBI-HSS \\
\hline $\begin{array}{l}\text { Akman et al., } \\
2016 \text { [26] (Turkey) }\end{array}$ & $\begin{array}{l}165 \text { nurses who had worked at least } 1 \text { month in pediatric } \\
\text { clinics, surgery clinics, PICU, NICU }\end{array}$ & $\begin{array}{l}\text { A descriptive, comparative, } \\
\text { correlational cross-sectional design }\end{array}$ & MBI-HSS \\
\hline $\begin{array}{l}\text { Alves and } \\
\text { Guirardello, } 2016 \\
\text { [27] (Brazil) }\end{array}$ & $\begin{array}{l}267 \text { nurses from } 15 \text { inpatient wards and three intensive care } \\
\text { units of two pediatric hospitals }\end{array}$ & Cross-sectional correlational design & EE-HSS \\
\hline $\begin{array}{l}\text { Amin et al., } 2015 \\
\text { [28] (India) }\end{array}$ & 129 nurses with at least 1-year experience working in NICU & Cross-sectional design & ProQOL5 \\
\hline $\begin{array}{l}\text { Aytekin, } 2013 \text { [29] } \\
\text { (Turkey) }\end{array}$ & 85 nurses working in two NICUs & $\begin{array}{l}\text { Descriptive and correlational study } \\
\text { (multi-methods) }\end{array}$ & MBI-HSS \\
\hline $\begin{array}{l}\text { Barr, } 2018[30] \\
\text { (Australia) }\end{array}$ & 142 nurses from four NICUs & Cross-sectional cohort study & ProQOL5 \\
\hline $\begin{array}{l}\text { Barr, } 2018[31] \\
\text { (Australia) }\end{array}$ & 140 nurses from four NICUs & Cross-sectional survey design & ProQOL5 \\
\hline $\begin{array}{l}\text { Berger et al., } 2015 \\
\text { [32] (USA) }\end{array}$ & $\begin{array}{l}239 \text { pediatric nurses working in a five-hospital system which } \\
\text { included an urban pediatric tertiary care teaching hospital }\end{array}$ & Cross-sectional survey design & ProQOL5 \\
\hline $\begin{array}{l}\text { Bilal et al., } 2017 \\
\text { [33] (Pakistan) }\end{array}$ & $\begin{array}{l}113 \text { pediatric nurses working in Punjab's largest state-run } \\
\text { hospital }\end{array}$ & Cross-sectional survey design & $\begin{array}{l}\text { Five items adapted from } \\
\text { the Camp scale (1994) }\end{array}$ \\
\hline $\begin{array}{l}\text { Bourbonnais } \\
\text { et al., } 1998 \text { [34] } \\
\text { (Canada) }\end{array}$ & $1891 ; 57$ pediatric nurses employed at six acute care hospitals & $\begin{array}{l}\text { Cross-sectional survey design (phase } \\
1 \text { of a longitudinal study) }\end{array}$ & $\begin{array}{l}\text { MBI; only separated out } \\
\text { EE }\end{array}$ \\
\hline $\begin{array}{l}\text { Branch and } \\
\text { Klinkenberg, } 2015 \\
\text { [35] (USA) }\end{array}$ & $\begin{array}{l}\text { 296; } 179 \text { pediatric nurses on ED, PICU, CICU, haem-onc, and } \\
\text { cardiology units }\end{array}$ & Cross-sectional survey design & ProQOL5 \\
\hline $\begin{array}{l}\text { Bursch et al., } \\
2018 \text { [36] (USA) }\end{array}$ & $\begin{array}{l}115 \text { nurses working in an urban children's hospital PICU or } \\
\text { NICU }\end{array}$ & Cross-sectional survey design & Abbreviated MBI \\
\hline $\begin{array}{l}\text { Czaja et al., } 2012 \\
\text { [37] (USA) }\end{array}$ & $\begin{array}{l}173 \text { nurses on general medical, surgical and oncology wards, } \\
\text { PICU, and ED at a tertiary care children's hospital }\end{array}$ & Cross-sectional survey design & MBI-HSS \\
\hline $\begin{array}{l}\text { Davis et al., } 2013 \\
\text { [38] (USA) }\end{array}$ & $\begin{array}{l}\text { 74, } 15 \text { pediatric oncology nurses from two major medical } \\
\text { centers }\end{array}$ & $\begin{array}{l}\text { Cross-sectional survey design } \\
\text { (observational, descriptive research } \\
\text { design) }\end{array}$ & $\mathrm{MBI}$ \\
\hline $\begin{array}{l}\text { Dos Santos Alves } \\
\text { et al., } 2017 \text { [39] } \\
\text { (Brazil) }\end{array}$ & $\begin{array}{l}267 \text { nurses, nursing technicians, and nursing assistants on } 15 \\
\text { in-patient units and three ICUs in two pediatric hospitals }\end{array}$ & Cross-sectional survey design & MBI: EE \\
\hline $\begin{array}{l}\text { Downey et al., } \\
1995 \text { [40] (USA) }\end{array}$ & $59 \mathrm{NICU}$ nurses from a single state & $\begin{array}{l}\text { Multi-methods design (surveys and } \\
\text { open-ended questions) }\end{array}$ & $\begin{array}{l}\text { Adaptation of Popoff and } \\
\text { Funkhouser's survey of } \\
\text { nurses }\end{array}$ \\
\hline $\begin{array}{l}\text { Duxbury et al., } \\
1984 \text { [41] (USA) }\end{array}$ & 283 nurses from 14 level 3 NICUs & Cross-sectional survey design & The Tedium Scale \\
\hline $\begin{array}{l}\text { Edmonds et al., } \\
2012[42] \\
\text { (Canada) }\end{array}$ & $\begin{array}{l}\text { 182; } 88 \text { pediatric oncology nurses from four major hospital } \\
\text { centers }\end{array}$ & Interventional, pre/post-design & $\mathrm{MBI}$ \\
\hline $\begin{array}{l}\text { Estabrooks et al., } \\
2011[43] \\
\text { (Canada) }\end{array}$ & 844 nurses from 32 units across eight pediatric hospitals & Cross-sectional survey design & MBI-GS \\
\hline $\begin{array}{l}\text { Faller et al., } 2011 \\
\text { [44] (USA) }\end{array}$ & $\begin{array}{l}\text { 976; } 117 \text { pediatric/neonatal travel nurses employed by a large } \\
\text { healthcare staffing company }\end{array}$ & Cross-sectional survey design & CBI \\
\hline $\begin{array}{l}\text { Favrod et al., } \\
2018[45] \\
\text { (Switzerland) }\end{array}$ & 213; 91 NICU nurses from two university hospitals & $\begin{array}{l}\text { Concurrent triangulation, mixed- } \\
\text { methods cross-sectional study }\end{array}$ & $\mathrm{MBI}$ \\
\hline $\begin{array}{l}\text { Gallagher and } \\
\text { Gormley, } 2009 \\
\text { [46] (USA) }\end{array}$ & 30 BMT nurses from a large pediatric medical center & $\begin{array}{l}\text { Descriptive non-experimental design } \\
\text { multi-method design (survey }+ \text { an } \\
\text { open-ended question) }\end{array}$ & $\mathrm{MBI}$ \\
\hline Gauthier et al., & $45 \mathrm{PICU}$ nurses at an urban pediatric academic hospital & Interventional, pre/post-design & MBI-HSS \\
\hline
\end{tabular}


Table 1 Characteristics of 78 studies exploring pediatric nurse burnout included in the scoping review (Continued)

\begin{tabular}{|c|c|}
\hline $\begin{array}{l}\text { Author(s), year, } \\
\text { country }\end{array}$ & Sample \\
\hline \multicolumn{2}{|l|}{2015 [47] (USA) } \\
\hline $\begin{array}{l}\text { Günüşen et al., } \\
2018 \text { [48] (Turkey) }\end{array}$ & $\begin{array}{l}117 \text { pediatric nurses caring for children with chronic illness at } \\
\text { an urban children's public hospital from critical care, CV } \\
\text { surgery, oncology, premature care, neonatal care, and burn } \\
\text { care }\end{array}$ \\
\hline $\begin{array}{l}\text { Habadi et al., } \\
2018 \text { [49] (Saudi } \\
\text { Arabia) }\end{array}$ & $\begin{array}{l}\text { 182; } 22 \text { pediatric nurses working in an academic hospital } \\
\text { including pediatric ward, NICU, and PICU }\end{array}$ \\
\hline $\begin{array}{l}\text { Hallberg, } 1994 \\
\text { [50] (Sweden) }\end{array}$ & $\begin{array}{l}11 \text { pediatric mental health nurses from a nine-bed child psy- } \\
\text { chiatric unit }\end{array}$ \\
\hline $\begin{array}{l}\text { Holden et al., } \\
2011 \text { [51] (USA) }\end{array}$ & $\begin{array}{l}347 \text { nurses at two urban academic tertiary care free-standing } \\
\text { pediatric hospitals and three inpatient units were studied at } \\
\text { each hospital: PICU hematology and oncology transplant and } \\
\text { general medical/surgical }\end{array}$ \\
\hline
\end{tabular}

Hsu et al., 2010

[52] (Taiwan)

Hylton Rushton et al., 2015 [53] (USA)

Jacobs et al., 2018 [54] (USA)

Kase et al., 2018

[55] (USA)

Klein et al., 2017

[56] (Switzerland)

Koivula et al.,

2000 [57]

(Finland)

Latimer et al. 2017 [58]

(Canada)

Lewiston et al., 1981 [59] (USA)

Li et al., 2014 [60] (USA)

Liakopoulou et al., 2008 [61] (Greece)

Lin et al., 2009

[62] (China)

Lin et al., 2016

[63] (Taiwan)

Liu et al., 2018

[64] (China)

Maytum et al., 2004 [65] (USA)

Meadors et al., 2009 [66] (USA)

Messmer et al., 2011 [67] (USA) general medical/surgica

121: five pediatric nurses found through the National Union of Nurses' Associations Republic of China database

114; 20 pediatric nurses from two pediatric/neonatal units

206; 47 nurses from six companies of the pediatric healthcare system and included employees from the medical center, primary care, home health, and other supporting departments in the system

150; 43 pediatric palliative care nurses identified through the American Academy of Pediatrics

398; 302 nurses from nine level 3 NICUs

723; 21 pediatric nurses from two hospitals

51; 27 female NICU and PICU nurses at large tertiary pediatric hospital

96; 38 nurses attending a workshop for health professionals who work with children with chronic illness

251 new nurses entering a residency program at an urban children's hospital

113; 71 nurses from two pediatric departments

249; 14 pediatric nurses working in a large public teaching hospital

144 female PICU nurses from seven regional teaching hospitals or higher-level medical institutions

$1761 ; 101$ nurses from nine public tertiary hospitals in four provinces

20 nurses recruited from throughout the state with extensive nursing expertise working with children with chronic conditions

167; 23 nurses located nationwide working in PICU, NICU, or general pediatrics

33 nurses with 2 years or less experience hired at a children's
Study design

Burnout measurement

tool

Mixed-methods design (cross-

$\mathrm{MBI}$

sectional survey + interviews)

Cross-sectional survey design

MBI-HSS

Interventional mixed-methods design $\mathrm{MBI}$ and the Tedium (supervisory sessions, surveys + open Measure ended questions)

Cross-sectional survey design

$M B I: E E$

Cross-sectional survey design

Occupational Burnout Scale

$\mathrm{MBI}$ hospital
Cross-sectional survey design

$\mathrm{MBI}$ and $\mathrm{CB}$

Cross-sectional survey design

Cross-sectional survey design

Cross-sectional survey design

Cross-sectional design (survey + fMRl)

Cross-sectional survey design

Cross-sectional survey design

Cross-sectional, comparative, and correlational descriptive design

Cross-sectional survey design

Cross-sectional, correlational, survey design

Cross-sectional survey design

Descriptive, qualitative study

Cross-sectional, correlational survey design

Cross-sectional descriptive, correlational study
The Compassion Fatigue and Satisfaction Self-Test for Helpers

A selection of 10 questions from the $\mathrm{MBI}$ covering each subscale

Paunonen's instrument

Compassion Fatigue Scale

$\mathrm{MBI}$

Compassion satisfaction and fatigue test

$\mathrm{MBI}$

MBI-HSS

Occupational Burnout Inventory

(Chinese) MBI-GS

Self-identification

ProQL

The Maslach Burnout Inventory (MBI) 
Table 1 Characteristics of 78 studies exploring pediatric nurse burnout included in the scoping review (Continued)

\begin{tabular}{|c|c|c|c|}
\hline $\begin{array}{l}\text { Author(s), year, } \\
\text { country }\end{array}$ & Sample & Study design & $\begin{array}{l}\text { Burnout measurement } \\
\text { tool }\end{array}$ \\
\hline $\begin{array}{l}\text { Meyer et al., } 2015 \\
\text { [16] (USA) }\end{array}$ & $\begin{array}{l}251 \text { nurses entering an RN residency program at an urban } \\
\text { children's hospital }\end{array}$ & Longitudinal study & $\begin{array}{l}\text { Compassion Fatigue Self- } \\
\text { Test }\end{array}$ \\
\hline $\begin{array}{l}\text { Moody et al., } \\
2013 \text { [68] (USA } \\
\text { and Israel) }\end{array}$ & $\begin{array}{l}\text { 47; } 25 \text { pediatric oncology nurses from two urban academic } \\
\text { pediatric hematology/oncology programs }\end{array}$ & $\begin{array}{l}\text { Interventional, mixed methods } \\
\text { (survey + journal), pre/post-design }\end{array}$ & $\mathrm{MBI}$ \\
\hline $\begin{array}{l}\text { Morelius et al., } \\
2013[69] \\
\text { (Sweden) }\end{array}$ & $\begin{array}{l}47 \text { nurses from two departments at a university hospital, a } \\
\text { level } 3 \mathrm{NICU} \text {, and a child and adolescent psychiatry inpatient } \\
\text { ward }\end{array}$ & An exploratory, prospective design & $\mathrm{CBI}$ \\
\hline $\begin{array}{l}\text { Morrison Wylde } \\
\text { et al., } 2017 \text { [70] } \\
\text { (USA) }\end{array}$ & $\begin{array}{l}95 \text { nurses entering a pediatric nurse residency program in a } \\
\text { children's hospital in an urban area }\end{array}$ & Interventional pre/post-design & $\begin{array}{l}\text { The Compassion Fatigue } \\
\text { Self-Test }\end{array}$ \\
\hline $\begin{array}{l}\text { Moussa and } \\
\text { Mahmood, } 2013 \\
\text { [71] (Egypt) }\end{array}$ & 55 PICU nurses & $\begin{array}{l}\text { Cross-sectional, descriptive, } \\
\text { correlational design }\end{array}$ & MBI-HSS \\
\hline $\begin{array}{l}\text { Mudallal et al., } \\
2017[72] \\
\text { (Jordan) }\end{array}$ & 407; 39 pediatric nurses, total sample from 11 hospitals & Cross-sectional, correlational design & MBI-HSS \\
\hline $\begin{array}{l}\text { Murphy-Oikonen } \\
\text { et al., } 2010 \text { [73] } \\
\text { (Canada) }\end{array}$ & 14 NICU nurses at a regional hospital & $\begin{array}{l}\text { Qualitative exploratory study; } \\
\text { computer-assisted personal interview } \\
\text { format }\end{array}$ & Self-identification \\
\hline $\begin{array}{l}\text { Neumann et al., } \\
2018 \text { [74] (USA) }\end{array}$ & 914; 238 pediatric hematopoietic cell transplant nurses & Cross-sectional survey design & $\mathrm{MBI}$ \\
\hline $\begin{array}{l}\text { Nguyen et al., } \\
2018[75] \\
\text { (Vietnam) }\end{array}$ & $\begin{array}{l}\text { 500; } 78 \text { pediatric nurses from a general hospital, children's } \\
\text { hospital and obstetric hospital }\end{array}$ & Cross-sectional survey design & MBI:GS \\
\hline $\begin{array}{l}\text { Oehler et al., } \\
1991 \text { [77] (USA) }\end{array}$ & $\begin{array}{l}49 \text { nurses in PICU, NICU, and intermediate care unit at a large } \\
\text { tertiary medical center }\end{array}$ & Cross-sectional survey design & $\mathrm{MBI}$ \\
\hline Oehler and & 121 nurses working in a level 3 NICU & Cross-sectional correlational design & $\mathrm{MBI}$ \\
\hline
\end{tabular}

Davidson, 1992

[76] (USA)

Ohue et al., $2011 \quad 336 ; 27$ pediatric nurses from three acute care hospitals [78] (Japan)

Pagel and Wittmann, 1986 [79] (USA)

Paula Vega et al. 2017 [98] (Chile)

Profit et al., 2014 [80] (USA)

Richter et al., 2012 [81] (South Africa)

Robins et al., 2009 [82] (USA)

314; 136 nurses from the Divisions of Oncology, Cardiology, Nephrology, Emergency Services, Intensive Care Units, Rehabilitation/Child Development, Psychology, Social Work, and Anesthesiology. General medical nurses who provided care on the medical/surgical and critical care floors but did not belong to a specific pediatric division were also recruited.

Rochefort and Clarke, 2010 [83] (Canada)

Rodrigues et al., 2018 [85] (USA)

339 NICU nurses working in nine large urban, publiclyadministered teaching hospitals

32 and 41; study 1: 32 nurses working in a 44-bed pediatric in- Mixed methods (focus groups +

patient care unit at a large urban hospital; study 2: participants surveys)

included 41 nurses working in general pediatric inpatient units at two large urban children's hospitals (27 from site 1 and 14 from site 2)

Rodrigues et al., 33 nurses working on a 44-bed pediatric inpatient care unit at Interventional, post-intervention,

\section{Cross-sectional survey design}

Multi-methods survey design

Cross-sectional design

Cross-sectional survey study

Interventional pre/post-design

Cross-sectional design

Cross-sectional correlational survey design
MBI (revised version)

The Tedium Measure

MBI

Four-item EE scale, based on the MBI

MBI-HSS

Compassion Satisfaction and Fatigue Test

MBI: EE

Study 1: self-report; study 2: MBI: EE, DP

MBI: EE, DP 
Table 1 Characteristics of 78 studies exploring pediatric nurse burnout included in the scoping review (Continued)

\begin{tabular}{|c|c|c|c|}
\hline $\begin{array}{l}\text { Author(s), year, } \\
\text { country }\end{array}$ & Sample & Study design & $\begin{array}{l}\text { Burnout measurement } \\
\text { tool }\end{array}$ \\
\hline 2018 [84] (USA) & a large urban hospital & repeated measures design & \\
\hline $\begin{array}{l}\text { Rodriguez-Rey } \\
\text { et al., } 2018 \text { [86] } \\
\text { (Spain) }\end{array}$ & $\begin{array}{l}\text { 487; } 281 \text { nurses from PICU and general pediatrics at nine } \\
\text { hospitals }\end{array}$ & Cross-sectional survey design & $\mathrm{MBI}$ \\
\hline $\begin{array}{l}\text { Roney and Acri, } \\
2018 \text { [87] (USA) }\end{array}$ & $\begin{array}{l}318 \text { nurses_current members of the Society of Pediatric } \\
\text { Nurses (SPN) }\end{array}$ & Cross-sectional survey design & ProQOL \\
\hline $\begin{array}{l}\text { Sekol and Kim, } \\
2014[88](\text { USA) }\end{array}$ & $\begin{array}{l}240 \text { nurses working in surgical, medical, critical care, and } \\
\text { hematology/oncology units of a children's hospital }\end{array}$ & Cross-sectional survey design & ProQOL5 \\
\hline $\begin{array}{l}\text { Skorobogatova } \\
\text { et al., } 2017 \text { [89] } \\
\text { (Lithuania) }\end{array}$ & $\begin{array}{l}94 \text { nurses working in NICUs of two tertiary care perinatology } \\
\text { centers }\end{array}$ & Cross-sectional survey design & MBI-HSS \\
\hline $\begin{array}{l}\text { Soroush et al., } \\
2016 \text { [90] (Iran) }\end{array}$ & $86 \mathrm{NICU}$ nurses from all educational hospitals in the region & $\begin{array}{l}\text { Cross-sectional, descriptive, survey } \\
\text { design }\end{array}$ & $\mathrm{MBI}$ \\
\hline $\begin{array}{l}\text { Squires et al., } \\
2013[91] \\
\text { (Canada) }\end{array}$ & 735 nurses from 15 children's hospitals & Cross-sectional survey design & MBI-GS \\
\hline $\begin{array}{l}\text { Stimpfel et al., } \\
2013 \text { [92] (USA) }\end{array}$ & $\begin{array}{l}3710 \mathrm{NICU}, \mathrm{PICU} \text {, newborn nursery, and general pediatrics } \\
\text { nurses from } 342 \text { hospitals }\end{array}$ & Cross-sectional survey design & MBI-HSS \\
\hline $\begin{array}{l}\text { Sun et al., } 2017 \\
\text { [93] (China) }\end{array}$ & $602 ; 51$ pediatric nurses from three hospitals & Cross-sectional survey design & MBI-HSS \\
\hline $\begin{array}{l}\text { Sun et al., } 1996 \\
\text { [94] (China) }\end{array}$ & 1 100; 277 pediatric nurses from multiple medical centers & Cross-sectional survey design & $\mathrm{MBI}$ \\
\hline $\begin{array}{l}\text { Tawfik et al., } 2017 \\
\text { [95] (USA) }\end{array}$ & $1934 ; 1374$ NICU nurses from 44 NICUs in the same state & Cross-sectional survey design & $\mathrm{MBI}$ \\
\hline $\begin{array}{l}\text { Tawfik et al., } 2017 \\
\text { [96] (USA) }\end{array}$ & $2073 ; 1464$ NICU nurses from 44 NICUs in the same state & Cross-sectional design & $\mathrm{MBI}$ \\
\hline $\begin{array}{l}\text { Vicentic et al., } \\
2016 \text { [97] (Serbia) }\end{array}$ & $\begin{array}{l}\text { 60; } 30 \text { nurses/technicians of children with CP; control group of } \\
30 \text { pediatric nurses/technicians, as caregivers of normally } \\
\text { developing, non-palsy children }\end{array}$ & Case-control study & MBI-HSS \\
\hline $\begin{array}{l}\text { Watson and Feld, } \\
1996 \text { [99] (New } \\
\text { Zealand) }\end{array}$ & 14 pediatric ward nurses & $\begin{array}{l}\text { Multi-methods design (surveys and } \\
\text { open-ended questions) }\end{array}$ & $\mathrm{MBI}$ \\
\hline $\begin{array}{l}\text { Yao et al., } 2018 \\
\text { [100] (China) }\end{array}$ & $860 ; 44$ pediatric nurses & Cross-sectional design & MBI-GS \\
\hline $\begin{array}{l}\text { Zanatta and } \\
\text { Lucca, } 2015 \text { [101] } \\
\text { (Brazil) }\end{array}$ & $188 ; 57$ pediatric nurses at a single institution & Cross-sectional design & MBI-HSS \\
\hline
\end{tabular}

Total sample size is provided, in addition to sample size of pediatric nurses, if pediatric nurses were a subgroup of a larger sample MBI Maslach Burnout Inventory; MBI-GS Maslach Burnout Inventory-General Survey; MBI-HSS Maslach Burnout Inventory-Human Services Survey; EE Emotional Exhaustion, a subscale of the Maslach Burnout Inventory; DP Depersonalization, a subscale of the Maslach Burnout Inventory; CBI Copenhagen Burnout Inventory; ProQOL Professional Quality of Life Scale; ProQOL5 Professional Quality of Life Scale, version 5; ED emergency department; PICU pediatric intensive care unit; NICU neonatal intensive care unit; $C I C U$ cardiovascular intensive care unit; Haem-onc hematology-oncology; BMT bone marrow transplant; $C P$ cerebral palsy

$45,48,53,61,62,72,75,77,78,84-86,89,90,94,97$ $99,100]$, and 16 reporting proportions and/or severity of those with scores indicating emotional exhaustion (e.g., low, moderate, high). The mean of the reported raw Emotional Exhaustion scores was 22.45 (SD =6.54) which indicates moderate burnout [22]. Out of the 14 studies reporting the proportion of respondents with scores indicating high emotional exhaustion, the mean proportion was $38.7 \%$. The mean of the reported raw Depersonalization scores $[25,29,42,45,47,48,53,61$, $62,72,75,77,78,84-86,89,90,94,97,99,100]$ was $6.95(\mathrm{SD}=3.38)$ which indicates moderate burnout [22].
The mean of the reported raw Personal Accomplishment scores $[25,29,42,45,47,48,53,61,62,72,75,77,78$, $86,89,90,94,97,99,100]$ was $29.15(\mathrm{SD}=11.48)$ which indicates high personal accomplishment [22]. The individual scores from the MBI and other measurement tools are reported for each study in Table 2 .

\section{Factors related to burnout}

Of the included studies, 47 (60\%) addressed factors associated with pediatric nurse burnout (Table 3). Factors related to pediatric nurse burnout were classified into the following categories: nurse demographics, work 
Table 2 Pediatric nurse burnout scores by tool

\begin{tabular}{|c|c|c|c|c|c|c|}
\hline Author(s) & $\begin{array}{l}\text { Number } \\
\text { of } \\
\text { pediatric } \\
\text { nurses }\end{array}$ & $\begin{array}{l}\text { Adaption to the } \\
\text { tool }\end{array}$ & $\begin{array}{l}\text { Additional/alternative } \\
\text { results }\end{array}$ & $\begin{array}{l}\text { Personal Accomplishment } \\
\text { subscale }\end{array}$ & $\begin{array}{l}\text { Depersonalization } \\
\text { Subscale }\end{array}$ & $\begin{array}{l}\text { Emotional Exhaustion } \\
\text { Subscale }\end{array}$ \\
\hline Adwan [25] & 120 & No & & $\begin{array}{l}\text { PA: min-max, 13-48, avg. } \\
38, S D=5.7 \text { (moderate) }\end{array}$ & $\begin{array}{l}\text { DP: } 0-19, \text { avg. } 5.8, \\
S D=4.7 \text { (low) }\end{array}$ & $\begin{array}{l}\text { EE: } 5-45 \text {, avg. } 21, S D= \\
8.58 \text { (moderate) }\end{array}$ \\
\hline $\begin{array}{l}\text { Akman et al. } \\
{[26]}\end{array}$ & 165 & No & & PA: high & DP: low & EE: low \\
\hline $\begin{array}{l}\text { Alves and } \\
\text { Guirardello [27] }\end{array}$ & 267 & Yes & EE only & & & $\begin{array}{l}\text { EE: } \min -\max , 9-39, \\
\text { avg. }=21.5, \mathrm{SD}=6 \\
\text { (moderate) }\end{array}$ \\
\hline $\begin{array}{l}\text { Aytekin et al. } \\
\text { [29] }\end{array}$ & 85 & No & & $\begin{array}{l}\text { PA: } \text { min-max, } 1-22, \text { avg. }= \\
11.43, S D=4.63\end{array}$ & $\begin{array}{l}\text { DP: } \min -\max , 0-13, \\
\text { avg. }=3.87, S D= \\
2.77\end{array}$ & $\begin{array}{l}\text { EE: } \min -\max , 3-27 \\
\text { avg. }=14.9, S D=5.53\end{array}$ \\
\hline $\begin{array}{l}\text { Bourbonnais } \\
\text { et al. [34] }\end{array}$ & 57 & Yes & Separated out EE only & & & $\begin{array}{l}\text { High EE: } 20(35 \%) \\
\text { Crude Prevalence Ratio: } \\
1.04\end{array}$ \\
\hline Czaja et al. [37] & 173 & No & & $46 \%$ low PA & 38\% had high DP & $45 \%$ had EE \\
\hline Davis et al. [38] & 15 & No & & $\begin{array}{l}\text { PA: } 6.67 \% \text { low, } 53.3 \% \\
\text { mod, } 40 \% \text { high }\end{array}$ & & \\
\hline $\begin{array}{l}\text { Dos Santos } \\
\text { Alves et al. [39] }\end{array}$ & 267 & No & & & & $\begin{array}{l}\text { Moderate EE: mean } 21.5 \\
(\mathrm{SD} \pm 6) \text {; high EE: } 27 \%\end{array}$ \\
\hline $\begin{array}{l}\text { Edmonds et al. } \\
\text { [42] }\end{array}$ & 88 & No & & $\begin{array}{l}\text { Pre-intervention: PA: } \\
\text { mean } 35.9(6.4), 30.7 \% \\
\text { low PA }\end{array}$ & $\begin{array}{l}\text { Pre-intervention: } \\
\text { DP: mean 6.1(4.8), } \\
\text { 25\% high }\end{array}$ & $\begin{array}{l}\text { Pre-intervention: EE: } \\
\text { mean } 22.3 \text { (9.7), 24.1\% } \\
\text { high } \\
\text { Post-intervention: EE } \\
\text { change: mean 2.2 (6.3) } \\
\text { Wilcoxon signed-rank } \\
0.007 \\
\text { 7-month post- } \\
\text { intervention: EE change: } \\
\text { mean 2.3 (8.6) Wilcoxon } \\
\text { signed-rank 0.004 }\end{array}$ \\
\hline $\begin{array}{l}\text { Favrod et al. } \\
{[45]}\end{array}$ & 91 & No & & $\begin{array}{l}\text { PA: mean } 31.6(5.5), \\
\text { high }=3.6 \% \text {, mod. }= \\
35.7 \% \text {, low }=60.7 \%\end{array}$ & $\begin{array}{l}\text { DP: mean } 4.8(4.1) \\
\text { low }=64.3 \% \\
\text { mod. }=29.8 \% \\
\text { high }=6.0 \%\end{array}$ & $\begin{array}{l}\text { EE: } \text { mean }=23(9.9) \\
\text { low }=31 \%, \text { mod }= \\
47.6 \%, \text { high }=21.4 \%\end{array}$ \\
\hline $\begin{array}{l}\text { Gallagher and } \\
\text { Gormley [46] }\end{array}$ & 30 & No & & $\begin{array}{l}\text { PA: low }=16.7 \% \text {, mod. }= \\
33.3 \% \text {, high }=50 \%\end{array}$ & $\begin{array}{l}\text { DP: low }=63.3 \% \text {, } \\
\text { mod. }=33.3 \% \text {, } \\
\text { high }=3.3 \%\end{array}$ & $\begin{array}{l}\text { EE: low }=26.7 \%, \text { mod. }= \\
46.7 \% \text {, High }=26.7 \%\end{array}$ \\
\hline $\begin{array}{l}\text { Gauthier et al. } \\
\text { [47] }\end{array}$ & 45 & No & $\begin{array}{l}\text { Pre/post-intervention } \\
\text { trends: significant } \\
\text { quadratic trajectory of PA } \\
\text { in which PA increased at } \\
\text { time } 2 \text { and decreased at } \\
\text { time } 3[F(1,35) 5.43, P= \\
0.03] \text {, with no significant } \\
\text { trends for EE and DP }\end{array}$ & $\begin{array}{l}\text { Baseline: high PA mean } \\
47.29 \text { (7.43) }\end{array}$ & $\begin{array}{l}\text { Baseline: High DP, } \\
\text { mean = } 13.33 \text { (5.84) }\end{array}$ & $\begin{array}{l}\text { Baseline: High } \mathbf{E E}, \\
\text { mean }=31.39(9.85)\end{array}$ \\
\hline $\begin{array}{l}\text { Günüşen et al. } \\
\text { [48] }\end{array}$ & 117 & No & & PA: 19.20 (4.18) & DP: 6.14 (2.98), & EE: $18.85(6.21)$ \\
\hline $\begin{array}{l}\text { Habadi et al. } \\
\text { [49] }\end{array}$ & 22 & No & Burnout $=0.55 \%$ & Low PA: $36.4 \%$ & High DP: $22.7 \%$ & High EE: 59\% \\
\hline $\begin{array}{l}\text { Hylton et al. } \\
{[53]}\end{array}$ & 20 & No & & EE: $39.9(7.1)$ & DP: $12.5(6.2)$ & EE: 33 (13.8) \\
\hline $\begin{array}{l}\text { Jacobs et al. } \\
{[54]}\end{array}$ & 47 & $\begin{array}{l}\text { Yes-used in } \\
\text { conjunction } \\
\text { with } \\
\text { Copenhagen } \\
\text { Burnout }\end{array}$ & $\begin{array}{l}\text { Analysis of subgroup } \\
\text { differences between } \\
\text { nurses and non-nurses in- } \\
\text { dicated a trend toward } \\
\text { nurses reporting higher }\end{array}$ & & & \\
\hline
\end{tabular}


Table 2 Pediatric nurse burnout scores by tool (Continued)

\begin{tabular}{|c|c|c|c|c|c|c|}
\hline & & Inventory & $\begin{array}{l}\text { work-related burnout } \\
\text { (39.92 vs. } 35.86, P<.067) \\
\text { and client-related burnout } \\
\text { ( } 23.88 \text { vs. } 18.96, P<.061 \text { ). }\end{array}$ & & & \\
\hline Klein et al. [56] & 302 & $\begin{array}{l}\text { Yes-selection } \\
\text { of } 10 \text { questions } \\
\text { of the Maslach } \\
\text { Burnout } \\
\text { Inventory: MBI } \\
\text { covering each } \\
\text { subscale }\end{array}$ & 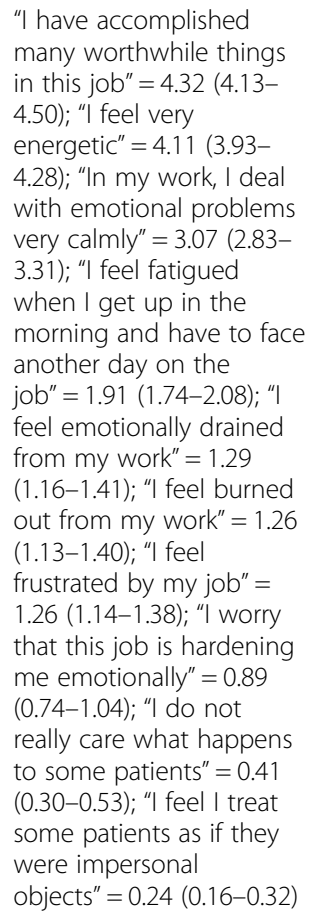 & & & \\
\hline $\begin{array}{l}\text { Lewiston et al. } \\
\text { [59] }\end{array}$ & 38 & No & $\begin{array}{l}9 \text { RNs had burnout } \\
\text { greater than the mean } \\
\text { (high burnout) and } 11 \\
\text { had burnout below the } \\
\text { mean (low burnout) }\end{array}$ & & & \\
\hline $\begin{array}{l}\text { Liakopoulou } \\
\text { et al. [61] }\end{array}$ & 71 & No & & PA mean $=37.8(5.8)$ & DP mean = $5.2(4.9)$ & EE mean $=27.5(9.5)$ \\
\hline Lin et al. [62] & 14 & No & & $\begin{array}{l}\text { PA mean }=34.5(7.1) \\
\text { (moderate) }\end{array}$ & $\begin{array}{l}\text { DP mean = } 3.6(4.0) \\
(\text { low })\end{array}$ & $\begin{array}{l}\text { EE mean = } 24.2(15.6) \\
\text { (moderate) }\end{array}$ \\
\hline Liu et al. [64] & 101 & No & $\begin{array}{l}\text { mean }=7.09 \text { (3.23) (lower } \\
\text { job burnout) }\end{array}$ & & & \\
\hline $\begin{array}{l}\text { Moussa and } \\
\text { Mahmood [71] }\end{array}$ & 55 & No & & $\begin{array}{l}\text { PA: high level burnout } \\
14.5 \% \text {, low level burnout } \\
85.5 \%\end{array}$ & $\begin{array}{l}\text { DP: high level } \\
\text { burnout } 74.5 \% \text {, low } \\
\text { level burnout } 25.5 \%\end{array}$ & $\begin{array}{l}\text { EE: high level burnout } \\
56.4 \% \text {, low level } \\
\text { burnout } 43.6 \% \text {; }\end{array}$ \\
\hline $\begin{array}{l}\text { Mudallal et al. } \\
\text { [72] }\end{array}$ & 39 & No & & PA: mean = $31.23(12.14)$ & $\begin{array}{l}\text { DP: } \text { mean }=12.74 \\
(4.89)\end{array}$ & EE: mean $=28.33(12.63)$ \\
\hline $\begin{array}{l}\text { Nguyen et al. } \\
{[75]}\end{array}$ & 78 & No & $\begin{array}{l}16.7 \% \text { burned out, } 14.1 \% \\
\text { exhausted, } 3.8 \% \\
\text { depressed, } 65.4 \% \text { healthy }\end{array}$ & $\mathbf{P A}=4.06(0.61)$ & $\begin{array}{l}\text { DP mean }=2.86 \\
(0.73)\end{array}$ & $\mathrm{EE}$ mean $=3.12(0.95)$ \\
\hline $\begin{array}{l}\text { Oehler and } \\
\text { Davidson [76] }\end{array}$ & 121 & No & $\begin{array}{l}\text { Overall, scores represent } \\
\text { moderate EE, DP, and } \\
\text { high lack of PA }\end{array}$ & $\begin{array}{l}\text { low PA mean }=36.6, \text { mod } \\
\text { PA mean }=32.8, \text { high PA } \\
\text { mean }=30.6\end{array}$ & $\begin{array}{l}\text { low } \mathbf{D P} \text { mean }=2.7 \\
\text { mod } \mathbf{D P} \text { mean }= \\
7.2, \text { high } \mathbf{D P} \\
\text { mean }=11.9\end{array}$ & $\begin{array}{l}\text { Low } \mathrm{EE} \text { mean }=12.2, \\
\text { mod } \mathrm{EE} \text { mean }=24.2, \\
\text { high } \mathrm{EE} \text { mean }=32.7\end{array}$ \\
\hline $\begin{array}{l}\text { Oehler et al. } \\
\text { [77] }\end{array}$ & 49 & No & & $\begin{array}{l}\text { PA mean = } 32.1 \text { (6.9) } \\
\text { (high) }\end{array}$ & $\begin{array}{l}\text { DP mean = } 6.9(4.9) \\
\text { (moderate) }\end{array}$ & $\begin{array}{l}\text { EE mean }=24.1(10.1) \\
\text { (moderate) }\end{array}$ \\
\hline Ohue et al. [78] & 27 & $\begin{array}{l}\text { Yes-revised } \\
\text { version of } \mathrm{MBI}\end{array}$ & & $\mathbf{P A}$ mean $=14.44(4.26)$ & $\begin{array}{l}\text { DP mean }=12.44 \\
(4.21)\end{array}$ & EE mean $=17.44(3.61)$ \\
\hline $\begin{array}{l}\text { Paula Vega } \\
\text { et al. [98] }\end{array}$ & 153 & No & & $\begin{array}{l}79.7 \%(n=122) \text { showed } \\
\text { medium to low level of } \\
\text { PA, and it was more }\end{array}$ & $\begin{array}{l}16 \%(n=11) \text { had a } \\
\text { high level of } \mathbf{D P} \text {. }\end{array}$ & $\begin{array}{l}48.4 \%(n=74) \text { of } \\
\text { participants had a } \\
\text { medium or high level of }\end{array}$ \\
\hline
\end{tabular}


Table 2 Pediatric nurse burnout scores by tool (Continued)

evident in oncology

professionals, as $41 \%$ of

them showed low PA.
Yes-four-item

Emotional

Exhaustion scale, based on the MBI

Richter et al. $17 \quad$ No

[81]
Nurses reporting burnout mean $=26.9 \%, \mathrm{SD}=11.4$, $P=0.0004$ (based on the authors determined cutoff)
Yes-only EE subscale

Clarke [83]

Rodrigues et al. 73 [85]

Rodrigues et al. 33 [84]

Rodriguez-Rey 281

et al. [86]

Skorobogatova 94 et al. [89]
Yes-only EE and DP subscales Yes-only EE and DP subscales

\begin{tabular}{|c|c|c|c|}
\hline $\begin{array}{l}\text { Soroush et al. } \\
\text { [90] }\end{array}$ & 86 & No & $\begin{array}{l}\text { Burnout mean }=46.2 \\
(12.5)(\text { mod) }\end{array}$ \\
\hline $\begin{array}{l}\text { Stimpfel et al. } \\
\text { [92] }\end{array}$ & 3710 & No & $\begin{array}{l}\text { Nurses who work } 8 \mathrm{~h} \\
\text { shifts: } 25 \% \text { burned out; } \\
\text { nurses who work } 12 \mathrm{~h} \\
\text { shifts: } 24 \% \text { burned out; } \\
\text { nurses who work }>13 \mathrm{~h} \\
\text { shifts: } 46 \% \text { burned out }\end{array}$ \\
\hline Sun et al. [93] & 51 & No & $\begin{array}{l}14(27.5 \%) \text { were positive } \\
\text { for burnout, and } 37 \\
(72.5 \%) \text { were negative for } \\
\text { burnout. }\end{array}$ \\
\hline
\end{tabular}

Sun et al. [94] $277 \quad$ No

Vicentic et al. $60 \quad$ No

[97]
No

No
PA mean $=33.88(2.77)$

Nurses/techs for children with CP: PA mean $=36.40$ (6.473) (30\% low risk, 40\% mod risk, 30\% high risk) Nurses/tech for children without CP: PA mean = 36.37 (11.180) (47\% low
Nurses in the pilot study experienced average levels of PA during the pilot phase of the study. No changes across the pre/post-intervention phases.

Nurses in the pilot study experienced low levels of DP and no changes across the pre/ post-intervention phases.

DP mean $=6.24$ (4.67) (20\% have high DP)

DP pre: 11.34 (4.66) DP post: 9.25 (3.23) 63\% reported high DP at baseline, and 3 months after the intervention, there were still 34\% reporting high DP.

PA mean $=37.74(6.88)$

DP mean $=6.41$ (4.56)

PA mean = 29.1 (10.12); low levels of self-esteen achievement (as components of professional $617 \%$, and were found in levels were found in $23.4 \%$ of respondents

PA mean $=22.6(5.4$ (strong lack of PA))

DP mean $=3.8$

(4.75): moderate levels of DP in 9.6\% and high levels in $12.8 \%$ of nurses

DP mean $=2.6(31)$ (weak)

EE mean $=21.28$ (mod))
Nurses in the pilot study experienced high levels of $\mathbf{E E}$ and no changes across the pre/postintervention phases.

35.7\% had EE scores higher than the published norms for medical personnel.

EE mean $=24.39$ (11.68) (46\% have high $\mathbf{E E}$ )

EE pre: 32.38 (11.29), EE post: 29.47 (10.52) $73 \%$ of our nurses reported high EE at baseline, and 3 months after the intervention, there were still $47 \%$ of urses reporting high EE.

EE mean $=21.74(9.17)$

EE mean $=14.4$ (7.91): overall, moderate $\mathbf{E E}$ was common in $41.5 \%$ and high in $9.6 \%$ of neonatal nurses.
DP mean $=10.97$ (1.67)

Nurses/techs for children with CP: DP mean $=4.31(5.594)$ (87\% low risk, 13\% high risk) Nurses/tech for $\begin{array}{ll}\text { Nurses/tech for } & \text { Nurses/tech for children } \\ \text { children without CP: } & \text { without CP: EE mean = }\end{array}$
Nurses/techs for children with CP: $\mathbf{E E}$ mean = 25.67 (15.043) (33\% low risk, $17 \%$ mod risk, $50 \%$ high risk Nurses/tech for children 
Table 2 Pediatric nurse burnout scores by tool (Continued)

\begin{tabular}{|c|c|c|c|c|c|}
\hline & & & $\begin{array}{l}\text { risk, } 30 \% \text { mod risk, 23\% } \\
\text { high risk) }\end{array}$ & $\begin{array}{l}\text { DP mean }=4.47 \\
(4.584)(83 \% \text { low } \\
\text { risk, } 17 \% \text { high risk })\end{array}$ & $\begin{array}{l}17.57 \text { (10.153)(53\% low } \\
\text { risk, 30\% mod risk, 17\% } \\
\text { high risk) }\end{array}$ \\
\hline $\begin{array}{l}\text { Watson and } \\
\text { Feld [99] }\end{array}$ & 14 & No & PA mean $=34.93(4.53)$ & $\begin{array}{l}\text { DP mean }=4.86 \\
(4.41)\end{array}$ & EE mean $=22.27(10.31)$ \\
\hline Yao et al. [100] & 44 & No & PA mean $=11.0(9.7)$ & DP mean $=7.0(6.1)$ & EE mean $=32.8(15.5)$ \\
\hline $\begin{array}{l}\text { Zanatta and } \\
\text { Lucca [101] }\end{array}$ & 57 & No & $\begin{array}{l}\text { PA: high } 24.6 \% \text {, mod } \\
52.6 \% \text {, low } 22.8 \%\end{array}$ & $\begin{array}{l}\text { DP: high } 29.8 \% \text {, } \\
\text { mod } 43.9 \% \text {, low } \\
26.3 \%\end{array}$ & $\begin{array}{l}\text { EE: high } 24.6 \% \text {, mod } \\
49.1 \% \text {, low } 26.3 \%\end{array}$ \\
\hline
\end{tabular}

Professional Quality of Life Scale

\begin{tabular}{|c|c|}
\hline Author(s) & Number of pediatric nurses \\
\hline Amin et al. [28] & 129 \\
\hline Barr [30] & 142 \\
\hline Berger et al. [32] & 239 \\
\hline $\begin{array}{l}\text { Branch and Klinkenberg } \\
\text { [35] }\end{array}$ & 179 \\
\hline Meadors et al. [66] & 23 \\
\hline Roney and Acri [87] & 318 \\
\hline
\end{tabular}

Sekol and Kim [88] $\quad 240$

Compassion Fatigue Self-Test

$\begin{array}{ll}\text { Author(s) } & \text { Number of pediatric nurses } \\ \text { Kase et al. [55] } & 43 \\ \text { Latimer et al. [58] } & 27 \\ \text { Li et al. [60] } & 251 \\ \text { Meyer et al. [16] } & 251 \\ \text { Morrison Wylde et al. [70] } & 95 \\ & \\ \text { Robins et al. [82] } & 136 \\ \text { The Tedium Measure } & \\ \text { Author(s) } & \text { Number of pediatric nurses } \\ \text { Pagel and Wittmann [79] } & 74\end{array}$

The Copenhagen Burnout Inventory

Author(s) Number of pediatric nurses

Faller et al. [44] $\quad 117$

Jacobs et al. [54] $\quad 47$

Morelius et al. [69]

47
Results

$\begin{array}{lll}\text { Low: } 26.4 \%(34) & \begin{array}{l}\text { Moderate: } 50.4 \% \\ (65)\end{array} & \text { High: } 23.3 \%(30) \\ \text { mean }=2.4, S D=.52 & & \\ \text { Low: } 23 \%(55) & \begin{array}{l}\text { Moderate: } 47.7 \% \\ (114)\end{array} & \text { High: } 29.3(70) \\ \text { Mean }=49.7(9.6) & & \\ \text { Mean }=14.82(4.33) & \end{array}$

The 25th percentile ranking for the Burnout subscale of the Professional Quality of Life (ProQOL) measure was 43.45 , the 50th percentile ranking was 49.22, and the 75th percentile was 56.92 .

The average score on the burnout subscale is 50 , which is higher than the 50th percentile ranking in this current study (slightly lower than average levels of burnout).

Surgical unit burnout $=24.5(5.1)$; medical unit burnout $=22.8(4.7)$; critical care unit burnout $=23.0$ (4.7); hematology/oncology unit burnout $=20.2$ (3.9)

Results

$4.7 \%$ prevalence

Mean $=23(10.8)$ (significantly higher than pediatric allied health providers)

Mean $=24.01(S D=11.67)$

Mean $=24.01(S D=11.67)$

Score after smartphone-delivered mindfulness: 22.37 (11.90)

Score after traditional delivered mindfulness: 26.14 (11.25)

Mean $=27.8$ (10.6) —extremely low risk

Results

The burnout scale mean for all subjects was 3.5855 with a standard deviation of .701. The scores ranged from 1.7142 to 5.4766 (signifies evidence of burnout).

Results

Half had high work-related burnout, and half had low work-related burnout.

Analysis of subgroup differences between nurses and non-nurses indicated a trend toward nurses reporting higher work-related burnout (39.92 vs. $35.86, P<.067)$ and patient-related burnout (23.88 vs. $18.96, P<.061)$. ${ }^{*}$ Conducted with $\mathrm{MBI}$

NICU nurses: $\mathrm{CBI}$, personal related burnout: 32.4 (13.8); work-related burnout: 24.3 (9.9); client-related burnout: 13.3 (9.1)

Child and adolescent psychiatry nurses: personal-related burnout: 32.4 
Table 2 Pediatric nurse burnout scores by tool (Continued)

(14.5); work-related burnout: 28.1 (16.3); client-related burnout: 22.9 (15.7)

Occupational Burnout Inventory

$\begin{array}{ll}\text { Author(s) } & \text { Number of pediatric nurses } \\ \text { Hsu et al. [52] } & 5 \\ \text { Jacobs et al. [54] } & 47\end{array}$

Results

Mean $=60.00(S D=11.75)$ (scale is out of 90 , higher = greater burnout)

Analysis of subgroup differences between nurses and non-nurses indicated a trend toward nurses reporting higher work-related burnout (39.92 vs. $35.86, P<.067)$ and patient-related burnout ( 23.88 vs. $18.96, P<.061)$.

Paunonen's instrument

Author(s) Number of pediatric nurses Results

Koivula et al. [57] 21

Mean $=6.15, \mathrm{SD}=(0.71)($ mild $)$

Popoff and Funkhouser's survey of nurses (adapted version)

Author(s) Number of pediatric nurses

Personal Accomplishment subscale

Downey et al. [40]

59

PA mean $=1.0(0.8)$

$M B I$ Maslach Burnout Inventory; EE Emotional Exhaustion, a subscale of the Maslach Burnout Inventory; DP Depersonalization, a subscale of the Maslach Burnout Inventory; PA Personal Accomplishment, a subscale of the Maslach Burnout Inventory; $C P$ cerebral palsy; ProQOL Professional Quality of Life Scale; $C B I$ Copenhagen Burnout Inventory; SD standard deviation, NICU neonatal intensive care; Mod moderate

environment, work attitudes, work outcomes, and burnout interventions.

\section{Nurse personal factors}

Burnout was found to be inversely associated with age; higher burnout was also associated with low/moderate level of experience (5-10 years) [26, 32, 46, 47, 58, 71, $85,88]$. A lack of university-level education or lower self-reported levels of clinical competency were also associated with higher levels of burnout $[57,90]$. Being in a nursing supervisory position had ambiguous results on impact on burnout; in some studies, holding supervisory positions correlated with higher reports of burnout while in others, the opposite effects were found [29, 33]. Nurses identifying as not being White or Asian/Pacific Islander ethnicity/race scored significantly lower on the MBI subscale of Personal Accomplishment than respondents identifying as White and Asian/Pacific Islander, and Asian/Pacific Islanders scored lower on Emotional Exhaustion than those identifying as White [36]. High neuroticism and low agreeableness [31] were both associated with higher burnout. Finally, being married had mixed results on impact on burnout, whereas in some studies, being married correlated negatively with burnout, and in others, it correlated positively [26, 36, 101].

\section{Work environment}

The work environment is defined by the conditions in which nurses work; it influences work attitudes and, in turn, work outcomes [103]. Burnout was found to be high in certain high-acuity pediatric units including emergency, medical/surgical, surgery, pediatric intensive care unit (PICU), and neonatal intensive care unit (NICU) [26, 32, 35, 36, 76, 88]. Davis et al. [38] found that adult oncology nurses had higher personal accomplishment than pediatric oncology nurses while Neumann et al. [74] found nurses who care for both pediatric and adult patients had lower emotional exhaustion than those who cared for adult patents only. Conversely, Sun et al. [94] found that nurses who worked in adult obstetrics and gynecology units had more burnout than nurses who worked in pediatric units; however, Ohue et al. [78] reported the inverse. Working in hematology/oncology [88] and unit-level factors such as workload [65, 85], number of assigned patients [26], increased number of admissions, understaffing, and shifts $>8 \mathrm{~h}$ were associated with increased burnout [51, 92, 95, 96]. Aytekin et al. [29] found working longer years in the NICU was associated with lower levels of personal accomplishment. Brusch et al. [36] found that nurses working exclusively day shifts had higher levels of depersonalization than those working night shifts or a mix of days and nights. Favrod et al. [45] found NICU nurses reported more traumatic stressors in their working environment.

Pediatric nursing workplaces with a strict structure of rules and regulations [33] or nurse leaders who valued structure over staff considerations [41] were found to have nurses with higher burnout. Nurses who had higher perceived organizational support had lower burnout [39]. Higher burnout was generally associated with systems issues such as unreasonable policies, staffing shortages, insurance frustrations, high volumes of paperwork [65], lack of nursing supplies [36], and lack of regular staff meetings [56]. The relationship between resources and facets of burnout was mixed: Rochefort and Clarke [83] found a negative relationship between nurses' emotional exhaustion and their rating of the adequacy of the resources available to them, while Gallagher and Gormley [46] found that even nurses who reported that 
Table 3 Factors associated with pediatric nurse burnout

\begin{tabular}{lll}
\hline Author(s) & $\begin{array}{l}\text { Number of } \\
\text { pediatric nurses }\end{array}$ & Factors associated with burnout \\
\hline Akman et al. [26] & 165 & $\begin{array}{l}\text { Higher EE scores associated with working in emergency and surgery, moderate in internal med, PICU, } \\
\text { NICU } \\
\end{array}$ \\
& & Low DP scores associated with working in PICU, moderate in all other units
\end{tabular}

High PA, high all units.

Lower level of burnout associated with a high level of job satisfaction, being married, increased age, and decreased number of assigned patients

Amin et al. [28] 129

Aytekin et al. [29] 85

Barr [30]

Barr [31]

Berger et al. [32]

Bilal et al. [33]

Branch and Klinkenberg [35]

Bursch et al. [36]

[43]

Favrod et al. [45]

9

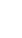

Higher burnout associated with greater perceived stress

Higher EE scores associated with being unhappy with their work environment

Lower EE associated with working at management level in NICU over other NICU nurses

Lower PA scores associated with longer years worked in the NICU

Core-self evaluations explained 33\% variance in burnout

Degree of agreeableness, neuroticism, extraversion, and positive affect contributed to variances in burnout Positive affect mediated the effect of core self-evaluations on burnout

Higher burnout associated with high neuroticism and low agreeableness and work stress, controlled for personality traits

Work stress mediated the effect of neuroticism and extraversion on burnout

Higher burnout and lower compassion satisfaction associated with nurses under 40 years of age, with 610 years of experience and/or working in a medical-surgical unit

Higher burnout associated with an organizational structure with rules and relations and being a supervisor Lower burnout and burnout prevention associated with participation in decision-making, instrumental communication, and promotional opportunities

Higher burnout associated with nursing in PICU over other units

Higher $\mathbf{E E}$ associated with nurses working most frequently in the PICU relative to those working most frequently in the NICU, those who found communication with nurses more stressful, and having a lack of necessary nursing supplies

Lower EE associated with being married or in a domestic partnership relative to respondents who were unmarried and not in a domestic partnership, identifying as Asian/Pacific Islander relative to respondents identifying as White

Higher DP associated with respondents working day shifts relative to those working the night shift or a mix of day and night shifts, nurses working most frequently in the PICU relative to those working most frequently in the NICU, greater endorsement of stress related to communication among nurses, the experience level of colleagues, staffing, and stress associated with the patient population

Lower DP associated with respondents working in the NICU relative to those working frequently in the PICU and respondents who reported being married or in a domestic partnership

Higher PA reported in individuals identifying as White and individuals identifying as Asian/Pacific Islander relative to individuals identifying as others

Lower PA found in nurses who found their own lack of knowledge, skills and/or confidence in themselves stressful and respondents identifying as being of another ethnicity/race relative to respondents identifying as White

Lack of burnout or PTSD associated with nurses who generally felt more positively about their work environment, with more confidence in their physician and nurse collogues as well as feeling a part of a team

Higher PA associated with working in adult oncology over pediatric oncology nurses

Lower burnout associated with nurses with a perception of having greater autonomy, greater control, good relationships at work, and organizational support, and are more satisfied with the work and the safety climate is assessed as more positive

Higher burnout found in staff nurses who have a head nurse with a leadership style of high structure and low consideration

Higher EE associated with lower job satisfaction

Similar burnout levels in NICU nurses and midwives 
Table 3 Factors associated with pediatric nurse burnout (Continued)

\begin{tabular}{|c|c|c|}
\hline Author(s) & $\begin{array}{l}\text { Number of } \\
\text { pediatric nurses }\end{array}$ & Factors associated with burnout \\
\hline & & NICU nurses more likely to reach the severe threshold of the three subscales of burnout than midwives \\
\hline & & NICU nurses reported more traumatic stressors in their working environment \\
\hline \multirow[t]{4}{*}{$\begin{array}{l}\text { Gallagher and } \\
\text { Gormley [46] }\end{array}$} & 30 & $\begin{array}{l}\text { Higher EE associated with higher DP and low PA, EE still present despite nurses reporting support } \\
\text { systems were in place and felt supported }\end{array}$ \\
\hline & & Lower EE associated with increased years as a BMT nurse \\
\hline & & Lower DP associated with increased years as a BMT nurse \\
\hline & & Higher PA associated with increased years as a BMT nurse \\
\hline \multirow[t]{5}{*}{ Gauthier et al. [47] } & 45 & Lower $\mathbf{E E}$ associated with self-compassion at time 1 and time 2, but not at time 3 \\
\hline & & Lower DP associated with elf-compassion at time 1 and time 2 , but not at time 3 \\
\hline & & Higher PA associated with self-compassion at all three time points \\
\hline & & All subscales of burnout were correlated with job satisfaction at time 1, but not at time 2 and time 3 \\
\hline & & $\begin{array}{l}\text { Lower burnout associated with more years of experience, job satisfaction had a significant positive } \\
\text { correlation with stress and burnout only at time } 1\end{array}$ \\
\hline \multirow[t]{2}{*}{ Holden et al. [51] } & 347 & $\begin{array}{l}\text { Higher burnout associated with unit-level staffing, task-level external mental workload, and job } \\
\text { dissatisfaction }\end{array}$ \\
\hline & & Burnout and job dissatisfaction were not significantly associated with the likelihood of medication error \\
\hline Klein et al. [56] & 302 & $\begin{array}{l}\text { Nurses rated lack of regular staff meetings, dissatisfaction with the quality of the decision-making process, } \\
\text { and providing futile treatment as significantly more stressful than physicians did }\end{array}$ \\
\hline Koivula et al. [57] & 21 & Higher burnout found in nurses with lower education level relative to those with higher education level \\
\hline Latimer et al. [58] & 27 & Higher burnout associated with nurses with less experience \\
\hline \multirow[t]{3}{*}{ Lewiston et al. [59] } & 38 & Higher EE associated with cystic fibrosis caregivers compared to controls \\
\hline & & Higher DP associated with cystic fibrosis caregivers compared to controls \\
\hline & & Equal PA from the job in cystic fibrosis caregivers and the control group \\
\hline \multirow[t]{2}{*}{ Lin et al. [63] } & 144 & $\begin{array}{l}\text { Higher burnout associated with higher work stress (after controlling for the demographics) and } \\
\text { depression }\end{array}$ \\
\hline & & $\begin{array}{l}\text { Occupational burnout had a mediating effect on the relationship between work stress and depression } \\
\text { levels }\end{array}$ \\
\hline
\end{tabular}

Factors associated with triggering burnout: seeing too many painful procedures done to children, seeing too much sadness, seeing too much death, angry, yelling families, and non-compliant patients/ families

Systems triggers: unreasonable policies, staffing shortages, insurance frustrations, paperwork, need to justify their position, and general healthcare system dysfunction

Role-specific triggers: lack of support, feeling you are on your own, less respondents cited unclear expectations, change in role and lack of challenge

Work overload: excessive demands of work

Personal triggers becoming overly involved or crossing professional boundaries

Factors associated with coping with burnout: short-term—self-care (exercise, meditation, journaling), fun/humor, non-work relationships; long-term personal coping strategies-developing a personal philosophy and faith and engaging in self-analysis

Short-term work-related coping strategies: developing supportive and honest professional relationships, need for their work to be congruent with their professional philosophy and interest

Lower burnout associated with nurses who would recommend their career to others relative to those who would recommend their career with reservation

Higher burnout predicated by current stress exposure after controlling for pre-existing stress exposure Lower burnout associated with "acting with awareness" at time 2

Higher EE associated with lack of access to work information

Lower $\mathbf{E E}$ associated with nurses increased age, length of professional experience, and experience on the 
Table 3 Factors associated with pediatric nurse burnout (Continued)

\begin{tabular}{|c|c|c|}
\hline Author(s) & $\begin{array}{l}\text { Number of } \\
\text { pediatric nurses }\end{array}$ & Factors associated with burnout \\
\hline & & unit \\
\hline & & $\begin{array}{l}\text { Lower DP associated with nurses increased age, length of professional experience, and experience on the } \\
\text { unit }\end{array}$ \\
\hline & & $\begin{array}{l}\text { Higher PA associated with nurses increased age, length of professional experience, and experience on the } \\
\text { unit }\end{array}$ \\
\hline $\begin{array}{l}\text { Murphy-Oikonen } \\
\text { et al. [73] }\end{array}$ & 14 & Higher burnout and frustration when caring for infants with neonatal abstinence syndrome \\
\hline Neumann et al. [74] & 238 & $\begin{array}{l}\text { Lower EE associated with caring for both pediatric and adult patients had lower relative to those who just } \\
\text { cared for adults only }\end{array}$ \\
\hline \multirow{2}{*}{$\begin{array}{l}\text { Oehler and } \\
\text { Davidson [76] }\end{array}$} & \multirow[t]{2}{*}{121} & Higher and more frequent burnout found in acute pediatric nurses relative to non-acute pediatric nurses \\
\hline & & $\begin{array}{l}\text { Higher burnout associated with increased job stress, workload, conflict with physicians, and uncertainty } \\
\text { regarding treatment }\end{array}$ \\
\hline \multirow[t]{3}{*}{ Oehler et al. [77] } & \multirow[t]{3}{*}{49} & $\begin{array}{l}\text { Higher EE predicted by job stress, trait anxiety, and experience on the current unit and explained } 55 \% \text { of } \\
\text { the variance }\end{array}$ \\
\hline & & Higher DP predicted by job stress and total work experience \\
\hline & & Lower PA predicted by level of supervisor support and state anxiety \\
\hline Ohue et al. [78] & 27 & $\begin{array}{l}\text { Higher PA found in nurses in the pediatrics and outpatient departments relative to those of the nurses in } \\
\text { the obstetrics and gynecology departments }\end{array}$ \\
\hline $\begin{array}{l}\text { Pagel and } \\
\text { Wittmann [79] }\end{array}$ & 74 & $\begin{array}{l}\text { Higher burnout related to higher reporting of the variable "percentage of children on a unit with social of } \\
\text { behavioral problems" }\end{array}$ \\
\hline $\begin{array}{l}\text { Rochefort and } \\
\text { Clarke [83] }\end{array}$ & 339 & Lower $\mathbf{E E}$ associated with higher ratings of nurse staffing and resource adequacy \\
\hline \multirow[t]{2}{*}{ Rodrigues et al. [85] } & \multirow[t]{2}{*}{73} & $\begin{array}{l}\text { Higher EE associated with greater time on the unit (moderate effect), nurses concern that current } \\
\text { standards of care inhibit optimal pain management, negative views of the hospital environment (large } \\
\text { effect), barriers to optimal pain management (moderate effect), lower self-efficacy (moderate effect), and } \\
\text { moral distress (moderate effect) }\end{array}$ \\
\hline & & $\begin{array}{l}\text { Burnout associated with expressions of exhaustion, frustration, overburden of their workload, and the } \\
\text { hopelessness in working with chronically ill pediatric patients, issues about self-efficacy regarding patient } \\
\text { outcomes }\end{array}$ \\
\hline \multirow[t]{2}{*}{ Sekol and Kim [88] } & \multirow[t]{2}{*}{240} & Higher burnout found in those with 5-9 years of experience working on the surgical unit \\
\hline & & $\begin{array}{l}\text { Lower burnout associated with working on the hematolgy/oncology unit, nursing experience of }>20 \\
\text { years, and all levels of experience if working on the hematolgy/oncology unit }\end{array}$ \\
\hline Soroush et al. [90] & 86 & Higher burnout associated with low clinical competency \\
\hline Squires et al. [91] & 735 & Higher DP associated with lower application of research information in the work context \\
\hline Stimpfel et al. [92] & 3710 & $\begin{array}{l}\text { Higher burnout associated with nurses who worked the longest shifts relative to those working shorter, 8- } \\
\text { h shifts }\end{array}$ \\
\hline Sun et al. [94] & 277 & $\begin{array}{l}\text { Higher burnout in nurses who worked in obstetrics and gynecology units relative to nurses who worked } \\
\text { in the surgery and pediatric units, in that order }\end{array}$ \\
\hline Tawfik et al. [95] & 1374 & Higher burnout associated with an average number of daily admissions of the NICU \\
\hline Tawfik et al. [96] & 1464 & Higher burnout in understaffed units \\
\hline Vicentic et al. [97] & 60 & $\begin{array}{l}\text { Higher } \mathbf{E E} \text { associated with higher anxiety and depression variables and higher risk of } \mathbf{E E} \text { for those who } \\
\text { care for children with CP than those who care for children without CP }\end{array}$ \\
\hline $\begin{array}{l}\text { Zanatta and Lucca } \\
\text { [101] }\end{array}$ & 57 & Higher EE associated with being married and having health problems related to work \\
\hline
\end{tabular}

EE Emotional Exhaustion, a subscale of the Maslach Burnout Inventory; DP Depersonalization, a subscale of the Maslach Burnout Inventory; PA Personal Accomplishment, a subscale of the Maslach Burnout Inventory; NICU neonatal intensive care unit; PICU pediatric intensive care unit; STS secondary traumatic stress; PTSD post-traumatic stress disorder; CP cerebral palsy

support systems were in place and felt supported still were emotionally exhausted. The lack of access to work information and research information was consistently associated with higher levels of burnout [71, 91], and lower burnout was associated with increased communication [33, 36] and better work relationships [39].

Factors impacting increased pediatric nurse burnout were related to the role of the nurse in patient care 
activities such as decision-making/uncertainty around treatment $[33,39,56,76]$, lack of role clarity, and unclear plan of care [65]. Other factors associated with the development of burnout were related to exposure to suffering, pain, sadness, and death [65]; hopelessness [85]; providing futile care [56]; and overall moral distress [85].

Higher levels of burnout were found in nurses who cared for specific pediatric patient populations such as caring for children with cerebral palsy [97], children with cystic fibrosis [59], and babies with neonatal abstinence syndrome [73]. Another patient factor related to higher burnout involved behavioral issues from patients/families $[65,79]$.

\section{Work attitudes}

Work attitudes are factors that impact the positive or negative perceptions of one's work environment [104].
Low self-compassion and low mindfulness [47] were associated with higher burnout. Co-occurring conditions with burnout such as depression [37, 63, 97], anxiety [97], and somatic work-related health problems [101] were correlated with greater burnout whereas positive psychosocial factors and coping strategies such as positive affect [30], acting with awareness [70], self-care, humor, reflection, non-work relationships, and a personal philosophy related to work were found to be associated with lower burnout [65].

Nurses' perceived work stress was positively associated with burnout in several studies [16, 28, 63, 76, 77]. Meyer et al. [16] found that current stress exposure significantly predicted higher levels of burnout after controlling for pre-existing stress exposure, and Holden et al. [51] found that burnout was positively associated with mental workload. Oehler and Davidson [76] found

Table 4 Burnout's relationship with other work outcomes in pediatric nurses

\begin{tabular}{lll}
\hline Author(s) & $\begin{array}{l}\text { Number of } \\
\text { pediatric nurses }\end{array}$ & Burnout outcomes \\
\hline $\begin{array}{l}\text { Alves and } \\
\text { Guirardello [27] }\end{array}$ & 267 & Higher EE associated with the outcome of a worse patient safety climate \\
$\begin{array}{ll}\text { Aytekin et al. [29] } \\
\text { Czaja et al. [37] }\end{array}$ & 173 & $\begin{array}{l}\text { Higher burnout associated with the outcome of decreased quality of life in the nurse } \\
\text { Higher burnout and PTSD were found in nurses considering a change of career, more frequently screened } \\
\text { positive for anxiety and depression, were more likely to respond negatively regarding their team } \\
\text { members, teamwork, and impact of their work }\end{array}$
\end{tabular}

A large portion of nurses with both burnout and significant PTSD symptoms found their symptoms interfered with their work and personal lives

Dos Santos Alves 267 et al. [39]

Günüşen et al. [48] 117

Latimer et al. [58] 27

Li et al. [60] 251

Lin et al. [63] 144

Meadors et al. [66] 23

Meyer et al. [16] 251
Lower burnout associated with the outcome of positive assessments of the safety climate

Secondary traumatic stress (STS) predicted $17 \%$ of EE

STS predicted $28 \%$ of DP

STS did not predict PA significantly

Higher burnout associated with nurses with higher secondary trauma

Higher likelihood of burnout with higher levels of PTSD

Burnout development secondary to PTSD symptoms may be mitigated by group cohesion

Higher burnout associated with the outcome of statistically significant influence on depression levels

Higher burnout associated with the outcome of higher levels of compassion fatigue

Higher burnout predicated by compassion fatigue after controlling for pre-existing stress exposure

Higher burnout predicated by current stress exposure after controlling for pre-existing stress exposure Mediated association: current stress exposure predicted higher levels of compassion fatigue which then predicted higher levels of burnout after 3 months of bedside

Exposure

Morrison Wylde $95 \quad$ Higher burnout associated with PTSD symptoms and compassion fatigue

et al. [70]

Moussa and

Mahmood [71]

55

Skorobogatova 94

et al. [89]

Tawfik et al. [96] 1464
Increased PA associated with the outcome of an increase in the mother's satisfaction with meeting the child's needs and expectations

Burnout associated with the symptom of tiredness

Higher burnout found in understaffed units with the outcome of higher infection rates during times when nurses feel overworked (likely when attention to infection prevention decreases)

EE Emotional Exhaustion, a subscale of the Maslach Burnout Inventory; PA Personal Accomplishment, a subscale of the Maslach Burnout Inventory; PTSD posttraumatic stress disorder 
perceived workload made a significant contribution to feelings of burnout. Job satisfaction was also found to be negatively associated with burnout $[26,29,39,43,47$, $51,67]$.

\section{Work outcomes}

Work outcomes refer to occupational performance factors that are influenced by work attitudes and the work environment [24]. Nine studies examined work outcomes associated with burnout including nurse retention, nurse well-being, patient safety, and patient-family satisfaction (Table 4). An increase in burnout was associated with nurses considering a career change [37], decreased quality of life [29], tiredness [89], and feeling negatively toward their teammates and the impact of their work [37]. Work-associated compassion fatigue $[16,66]$, secondary traumatic stress $[48,58]$, and posttraumatic stress disorder (PTSD) [37, 60, 70] were all found to be associated with pediatric nurse burnout. However, Li et al. [60] report that high group cohesion may prevent pediatric nurses from developing burnout from PTSD by protecting nurses from the impacts of negative outcomes. Nurse burnout was found to be negatively associated with the safety climate of the hospital in which they work [27, 39] and positively associated with higher infection rates when nurses were feeling overworked [96]. Moussa and Mahmood [71] found that as nurses' personal accomplishment increased, so did patients' mothers' satisfaction with meeting their child's care needs in the hospital.

\section{Burnout interventions}

Seven of the 78 studies included interventions to mitigate burnout (Table 5). Interventions included coping workshops [42], mindfulness activities [47, 68, 70], workshops to improve knowledge/understanding of their patient population [81, 85], and clinical supervision [50]. Only three of the seven interventions studied provided varying positive impacts on burnout scores [42, 70, 85]. An in-person day-long retreat resulted in a significant improvement in emotional exhaustion for pediatric nurses. The intervention involved didactic and hands-on trauma, adaptive grief, and coping strategies; half of the subjects were also randomized to a booster session 6 months later [42]. Another intervention involved a 90min interactive module on clinical skills surrounding the management of pediatric pain and resulted in a significant decrease in emotional exhaustion and depersonalization [85]. Finally, the third study of smartphone-delivered mindfulness interventions showed a marginal decrease in burnout compared to nurses receiving traditional mindfulness interventions [70].

\section{Discussion}

To our knowledge, this is the first scoping review that focuses on what is known about pediatric nurse burnout. Burnout was measured with a variety of instruments and interpretations, thereby making score comparisons a challenge. Even in those studies that used the MBI, the most commonly used burnout measurement [105], variations of the tool were applied, as were diverse subscale cutoff scores. Similar challenges in synthesizing extremely heterogeneous burnout data were echoed in a 2018 JAMA review of the prevalence of burnout among different types of physicians [106]. Of the MBI Emotional Exhaustion and Depersonalization subscale results that were synthesized, the results showed moderate scores indicating a significant level of burnout in pediatric nurses. Personal Accomplishment subscale results were high, perhaps indicating a factor of pediatric nursing that increases resilience despite moderate burnout in other domains. In studies that compared nurses who work in pediatric units to other in-patient units, burnout results were mixed [26, 32, 35, 36, 38, 74, 78, 94]. The majority of the included studies identified correlational relationships using cross-sectional study designs, which limited causal inferences. Study designs, such as longitudinal approaches, would allow for causal inference and in-depth analysis of this phenomenon in this unique population.

\section{Nurse personal factors}

Pediatric nurse demographic factors that are associated with burnout, such as age, work experience, and level of education, have been a common area of studied burnout associations across other healthcare populations. Similar burnout associations were found in research studying healthcare providers caring for adults such as younger age $(<31$ years) $[107,108]$ and years of experience $(>7$ years) [109]. It is likely that nurses new to the profession are younger, are experiencing the challenges of the nursing profession for the first time, and are less likely to have well-developed skills for resiliency. Given that the start of nurses' careers is a vulnerable stage for burnout, nursing schools and orientation programs may be wellpositioned to highlight burnout prevention and mitigation strategies with students and new hires [107]. Personality traits such as high neuroticism and low agreeableness were found to be associated with pediatric nurse burnout [31]. These results have been supported in other nurse and physician populations, along with conscientiousness, extraversion, and openness contributing to lower levels of burnout [110-113]. Although personality traits appear to have significant correlations with healthcare provider burnout, targeting modulation of personality traits as a mitigation strategy for burnout may be a high-cost, low-yield strategy. 
Table 5 Interventions for pediatric nurse burnout

\begin{tabular}{|c|c|c|c|}
\hline Author(s) & $\begin{array}{l}\text { Number of } \\
\text { pediatric } \\
\text { nurses }\end{array}$ & Type of intervention & Result \\
\hline $\begin{array}{l}\text { Edmonds } \\
\text { et al. [42] }\end{array}$ & 88 & $\begin{array}{l}\text { Care for the Professional Caregiver Program (CPCP): day-long re- } \\
\text { treat, includes didactic and discussion-based coverage of vicarious } \\
\text { trauma, loss and adaptive coping with grief. Practical, group-based } \\
\text { practice of coping strategies presented such as guided imagery, relax- } \\
\text { ation, body movement, and mindful breathing techniques that have } \\
\text { been adapted for the workplace. Half of the subjects were randomly } \\
\text { assigned to a booster session } 6 \text { months later. }\end{array}$ & $\begin{array}{l}\text { Pediatric nurses showed greatest improvement in the group in } \mathbf{E E} \\
\text { scores } 1 \text { month post-intervention and } 7 \text { months post-intervention. } \\
\text { results not impacted by receiving booster session or not. }\end{array}$ \\
\hline $\begin{array}{l}\text { Gauthier } \\
\text { et al. [47] }\end{array}$ & 45 & $\begin{array}{l}\text { 5-min daily mindfulness sessions. Conducted on the unit, as a group, } \\
\text { facilitated by a mindfulness meditation instructor. Mindfulness CDs and } \\
\text { booklets were distributed after the 1-month follow-up surveys were } \\
\text { completed. }\end{array}$ & $\begin{array}{l}\text { 1) Intervention was found to be feasible for PICU nurses. } \\
\text { 2) } \mathrm{EE} \text { was negatively correlated with mindfulness at all three time } \\
\text { points. } \\
\text { 3) PA was positively correlated with mindfulness at all three time } \\
\text { points. } \\
\text { 4) DP was not correlated with mindfulness at time } 3 \text { but was } \\
\text { negatively correlated with mindfulness at times } 1 \text { and } 2 \text {. }\end{array}$ \\
\hline
\end{tabular}

\begin{tabular}{|c|c|c|}
\hline $\begin{array}{l}\text { Hallberg } \\
{[50]}\end{array}$ & 11 & $\begin{array}{l}\text { Systematic group clinical supervision was performed every third week } \\
\text { for two full hours ( } 14 \text { sessions } / 28 \mathrm{~h} \text { all together. Supervision performed } \\
\text { by a registered nurse, with advanced training and extensive } \\
\text { experience in psychiatric care. }\end{array}$ \\
\hline
\end{tabular}

Moody $25 \quad$ Mindfulness-based course (MBC): 8 weeks of didactic and et al. [68] experiential mindfulness education via a structured, skills-training course delivered in a group setting at their hospital.; designed and facilitated at each site by a team of two licensed clinicians with extensive training and experience; included journaling.

\begin{tabular}{|c|c|c|}
\hline $\begin{array}{l}\text { Morrison } \\
\text { Wylde } \\
\text { et al. [70] }\end{array}$ & 95 & $\begin{array}{l}\text { Traditionally delivered mindfulness (TDM): Nurses in the TDM } \\
\text { intervention group (September 2013) received one group session per } \\
\text { week for } 4 \text { weeks led by a trained Buddhist Priest, taught within } \\
\text { different activities. Participants were encouraged to practice } \\
\text { mindfulness at other times during the day, but it was not mandatory } \\
\text { or assigned as homework. Smartphone-delivered mindfulness } \\
\text { (SDM): Nurses in the SDM received a free 3-month subscription to a } \\
\text { guided mindfulness meditation platform available via website or } \\
\text { smartphone application. }\end{array}$ \\
\hline $\begin{array}{l}\text { Richter } \\
\text { et al. [81] }\end{array}$ & 17 & $\begin{array}{l}\text { Nurses helped in the development of intervention materials. } \\
\text { Intervention package included five, short educational videos created to } \\
\text { demonstrate to nursing staff and caregivers' solutions to difficulties in } \\
\text { caring for hospitalized children affected by HIV/AIDS. Sessions run } \\
\text { every } 2 \text { weeks. }\end{array}$ \\
\hline
\end{tabular}

Rodrigues $33 \quad$ Nursing know-how: skills in working with pediatric chronic pain: et al. [84] 90-min group session developed from previous knowledge needs assessment (Rodrigues et al. 2017). Modules contained education and case-based role play using nurse's real experiences.
The mean score of the tedium degree decreased over the 12 months significantly for mental exhaustion. There were no significant changes in the degree of burnout as measured by the MBI.

No significant differences between the groups at baseline or at followup on the MBI.

SDM group reported significantly more "acting with awareness" and marginally more "non-reactivity to inner experience" skills compared to the TDM group. The SDM group showed marginally more compassion satisfaction and marginally less burnout. The SDM group had a lower risk for compassion fatigue compared to the TDM group, but only when the nurses had previous sub-clinical post-traumatic symptoms.

No changes in nurse well-being were found across the pre/post-intervention phases.

Post-intervention, patient mothers rated nurses as more supportive: mother-child interaction during feeding was more relaxed and engaged, babies were less socially withdrawn.

Significant improvements on both indicators of burnout-EE and DP - over the 3-month period. However, the proportion of nurses with high $\mathbf{E E}$ and $\mathbf{D P}$ is still high.

\section{EE Emotional Exhaustion, a subscale of the Maslach Burnout Inventory; DP Depersonalization, a subscale of the Maslach Burnout Inventory; PA Personal} Accomplishment, a subscale of the Maslach Burnout Inventory; MBI Maslach Burnout Inventory; TDM traditionally delivered mindfulness; SDM smartphonedelivered mindfulness; $M B C$ mindfulness-based course

It has been suggested that healthcare provider burnout is not a failure on the part of the individual, rather it is a culmination of impacts stemming from the work environment and the healthcare system as a whole [114]. Responsibility, then, is thought to lie within the individual, the organization, and the profession in general.

\section{Work environment}

Job demands and resource variables in pediatric nursing lead to increased burnout, work-life interference, psychosomatic complaints, and intent to leave; these associations are also represented in adult nursing literature [102, 115], including associations with excessive workload, number of assigned patients, admissions, understaffing, and longer shifts [116-119]. Although Bursch et al. [36] found that pediatric nurses who worked straight day shifts had higher depersonalization than those who worked mixed shifts or just night shifts, Poncet et al. [108] found that working more night shifts was associated with higher burnout in adult critical care nurses. Day shift nurses have potentially more strenuous workloads as patients are more wakeful, have diagnostic tests, or consulting services visiting; however, night shifts could be perceived as more strenuous as it requires the provider to work against their natural circadian rhythm and less support staff are available [120]. These results may also be dependent on individuals' preference and the specific unit on which they work.

Systems issues such as overwhelming clerical work, administrative, and resource issues have impacts on provider burnout in both the pediatric nurse and general physician populations $[36,56,65,121]$. Poor leadership is associated with pediatric nurse burnout as identified by Bilial and Ahmed [33] and Druxbury et al. [41]; this 
relationship is echoed in research with physicians, nurses, and allied health [122]. In pediatric nurses [39], increased perception of organizational support is associated with lower burnout; this association is supported in general nursing populations $[119,123]$. In all populations, the support a healthcare provider perceives they get from the organization is predictive of their level of organizational commitment. When healthcare providers perceive that they have high organizational support, they will exhibit greater organizational citizenship behavior, which are extra-role tasks that ultimately improve the organization [124]. Burnout itself results in reduced organizational commitment on the part of the healthcare provider [125].

The experience of witnessing patient suffering and death $[65,122]$, uncertainty around plan/utility of care $[15,56,126]$, moral distress $[15,85]$, and behavioral issues with patient families (e.g., aggressive patients/families) $[65,79,127]$ were found to be significant factors that contributed to burnout in both pediatric nurses and general population physicians and nurses.

\section{Work attitudes}

As might be expected, optimism, self-efficiency, resilience, and positive coping strategies are supported as inversely related to burnout in broader nursing populations [128-130]. The identification and treatment of burnout is particularly important to consider in light of the evidence that burnout is inversely related to job satisfaction and burnout is a contagious phenomenon between nurses; therefore, early intervention is essential to prevent transmission among staff [131-133].

\section{Work outcomes}

The association between burnout and patient satisfaction and intent to leave has been reported in non-pediatric nurse populations as well $[5,6,115,134-136]$. It is likely that as nurses become increasingly burned out their satisfaction with their jobs decreases and their desire to leave their position increases. This linkage highlights the importance of addressing nurse burnout in the organization to retain staff and reduce the financial and tacit knowledge losses associated with high nurse turnover.

Higher work-related burnout is also associated with mental health conditions such as anxiety and depression in pediatric nurses; this is represented in several studies of other healthcare provider populations [113, 137-140]. However, the majority of these associations are correlational; thus, they are left open for further assessment if they impact the development of burnout or if burnout impacted their development. Further research is needed to confirm causal, directional effects.
The relationship of increased clinician burnout and decreased patient safety has been supported in additional studies of healthcare provider burnout [7, 141]. As clinician burnout increases, the detachment from patients and their work does too, which may contribute to negative attitudes toward patient safety, incomplete infection control practices, and decreased patient engagement [7, 141]. Reducing burnout has the potential to impact patient safety; the Quadruple Aim of Healthcare hopes to improve patient outcomes, such as safety, through the addition of clinician well-being as a primary aim in the model [142].

\section{Interventions}

Although only seven of the studies analyzed in this review included interventions, there is modest evidence on the efficacy of burnout interventions in the broader healthcare provider population. Similar to the results of Hallberg [50], a study of Swedish district nurses showed no impact of clinical supervision on burnout [143]. While Morrison Wylde et al. [70] found a marginal improvement in pediatric nurse burnout with smartphonebased intervention vs. traditional mindfulness interventions, studies investigating mindfulness interventions in other healthcare populations reported mixed results [144-147]. Similar to pediatric nurses [84], social workers showed a significant decrease in burnout after attending skills development courses [148] suggesting that improving clinical knowledge and skills may reduce burnout. This is supported by the finding that pediatric nurses with lower clinical competency and education level have increased burnout [57, 90]. Although Edmonds et al. [42] showed significant decreases in pediatric nurse burnout using in-person trauma, adaptive grief, and coping sessions with follow-up, similar sessions have shown mixed results in other healthcare provider populations [149-151]. More research is needed to identify reliable interventions for pediatric nurse burnout that can be pre-emptively and routinely implemented by nursing schools and healthcare organizations.

\section{Study limitations}

The search strategy was limited to publications in English; thus, potentially relevant studies in other languages were excluded. Gray literature was not included; thus, informal annual surveys conducted at various healthcare institutions may have been missed; however, this was outweighed by the desire to only include peer-reviewed literature to ensure the quality of data reviewed [152]. Third, the definition of "nurses" varies internationally as does their required education and scope of practice; however, the slight variations were outweighed by the need to include thorough, culturally diverse research. 
Finally, the extreme heterogeneity of the burnout measurement tools and their application and interpretation inhibited the comparison of results across studies.

\section{Conclusion}

Our scoping review showed inconsistent measurement and interpretation of pediatric nurse burnout scores. Factors associated with pediatric nurse burnout were similar to those found in other healthcare professional groups and can be separated into the domains of nurse personal factors, work environment, work attitudes, and work outcomes. Only 45 of the 78 studies reviewed studied exclusive populations of pediatric nurses, and most associations identified were correlational. Few interventions to prevent or mitigate pediatric nurse burnout have been undertaken, and the results were mixed, at best. Further studies using mixed methods are needed to expand on these results and incorporate the direct feedback of the nurses. Additional research is needed to develop and test interventions for pediatric nurse burnout. The improvement of pediatric nurse burnout has the potential to improve nurse well-being and, ultimately, patient care.

\section{Abbreviations}

MBI: Maslach Burnout Inventory; NICU: Neonatal intensive care unit; PICU: Pediatric intensive care unit; PRISMA: Preferred Reporting Items for Systematic Reviews and Meta-Analyses; PTSD: Post-traumatic stress disorder

\section{Acknowledgements}

The authors would like to acknowledge librarian Mikaela Gray for her assistance with the search strategy development. Kristin Cleverley was supported by the CAMH Chair in Mental Health Nursing Research while writing this article.

\section{Authors' contributions}

LB was involved in the study design, data collection, data analysis, data interpretation, and drafting and finalizing the manuscript. WB and KC were involved in data interpretation and substantively revised the manuscript for important intellectual content. CM was involved in the data collection (title, abstract, and full-text screening) and substantively revised the manuscript for important intellectual content. KW was involved in the study design, data interpretation, and substantively revised the manuscript for important intellectual content. All authors read and approved the final manuscript and agree both to be personally accountable for their own contributions and to ensure that questions related to the accuracy or integrity of any part of the work are appropriately investigated, resolved, and the resolution documented in the literature. None of the authors have any competing interests as outlined by BioMed Central.

\section{Funding}

This paper is part of the doctoral work of the primary author who is funded by the Lawrence S. Bloomberg Faculty of Nursing, University of Toronto.

\section{Availability of data and materials}

The complete list of articles used as data in this review is available from the corresponding author on reasonable request.

Ethics approval and consent to participate

Not applicable.

Consent for publication

Not applicable.

\section{Competing interests}

The authors declare that they have no competing interests.

\begin{abstract}
Author details
${ }^{1}$ Lawrence S. Bloomberg Faculty of Nursing, University of Toronto, 155 College Street, Toronto, ON M5T 3M6, Canada. ${ }^{2}$ Hospital for Sick Children, 555 University Avenue, Toronto, ON M5G 1X8, Canada. ${ }^{3}$ Institute for Health Policy, Management and Evaluation, University of Toronto, 155 College Street, Toronto, ON M5T 3M6, Canada. ${ }^{4}$ Margaret and Wallace McCain Centre for Child, Youth and Family Mental Health, Centre for Addiction and Mental Health, Toronto, ON M5T 1R8, Canada.
\end{abstract}

Received: 26 August 2019 Accepted: 27 January 2020

Published online: 11 February 2020

\section{References}

1. Leiter MP, Maslach C, Schaufeli WB. Burnout: 35 years of research and practice. Career Dev Int. 2009:14(3):204-20.

2. Maslach C, Jackson SE. The measurement of experienced burnout. J Organ Behav. 1981:2(2):99-113.

3. Wang S, Yao L, Li S, Liu Y, Wang H, Sun Y. Sharps injuries and job burnout: a cross-sectional study among nurses in China. Nurs Health Sci. 2012;14(3): 332-8.

4. Han SS, Han JW, An YS, Lim SH. Effects of role stress on nurses' turnover intentions: the mediating effects of organizational commitment and burnout. Jpn J Nurs Sci. 2015;12(4):287-96.

5. Shanafelt TD, Noseworthy JH. Executive leadership and physician wellbeing: nine organizational strategies to promote engagement and reduce burnout. Mayo Clin Proc. 2017;92(1):129-46.

6. Vahey DC, Aiken LH, Sloane DM, Clarke SP, Vargas D. Nurse burnout and patient satisfaction. Med Care. 2004;42(2 Suppl):1157-66.

7. Cimiotti JP, Aiken LH, Sloane DM, Wu ES. Nurse staffing, burnout, and health care-associated infection. Am J Infect Control. 2012;40(6):486-90 [erratum appears in am J infect control. 2012 Sep;40(7):680].

8. Manomenidis G, Panagopoulou E, Montgomery A. Job burnout reduces hand hygiene compliance among nursing staff. J Patient Saf. 2017;13:13.

9. Johnson J, Louch G, Dunning A, Johnson O, Grange A, Reynolds C, et al. Burnout mediates the association between depression and patient safety perceptions: a cross-sectional study in hospital nurses. J Adv Nurs. 2017; 73(7):1667-80

10. Chiu S-F, Tsai M-C. Relationships among burnout, job involvement, and organizational citizenship behavior. J Psychol. 2006;140(6):517-30.

11. Zhou Y, Lu J, Liu X, Zhang P, Chen W. Effects of core self-evaluations on the job burnout of nurses: the mediator of organizational commitment. PLoS One. 2014;9(4):e95975.

12. Gabel Shemueli R, Dolan SL, Suarez Ceretti A, Nunez Del Prado P. Burnout and engagement as mediators in the relationship between work characteristics and turnover intentions across two Ibero-American nations. Stress Health. 2016:32(5):597-606.

13. Khamisa N, Peltzer K, Oldenburg B. Burnout in relation to specific contributing factors and health outcomes among nurses: a systematic review. Int J Environ Res Public Health. 2013;10(6):2214-40.

14. Nowrouzi B, Lightfoot $N$, Larivière $M$, Carter L, Rukholm E, Schinke R, et al. Occupational stress management and burnout interventions in nursing and their implications for healthy work environments: a literature review. Workplace Health Saf. 2015;63(7):308-15.

15. Larson CP, Dryden-Palmer KD, Gibbons C, Parshuram CS. Moral distress in PICU and neonatal ICU practitioners: a cross-sectional evaluation. Pediatr Crit Care Med. 2017;18(8):e318-e26.

16. Meyer RM, Li A, Klaristenfeld J, Gold Jl. Pediatric novice nurses: examining compassion fatigue as a mediator between stress exposure and compassion satisfaction, burnout, and job satisfaction. J Pediatr Nurs. 2015; 30(1):174-83.

17. Pradas-Hernandez L, Ariza T, Gomez-Urquiza JL, Albendin-Garcia L, De la Fuente El, Canadas-De la Fuente GA. Prevalence of burnout in paediatric nurses: a systematic review and meta-analysis. PLoS One. 2018;13(4): e0195039.

18. Tricco AC, Lillie E, Zarin W, O'Brien KK, Colquhoun H, Levac D, et al. PRISMA extension for scoping reviews (PRISMA-SCR): checklist and explanation. The PRISMA-ScR statement. Ann Intern Med. 2018;169(7):467-73. 
19. Hardin AP, Hackell JM. Age limit of pediatrics. Pediatrics. 2017;140(3): e20172151.

20. Li G, Abbade LPF, Nwosu I, Jin Y, Leenus A, Maaz M, et al. A scoping review of comparisons between abstracts and full reports in primary biomedical research. BMC Med Res Methodol. 2017;17(1):181.

21. Bramer WM, Giustini D, de Jonge GB, Holland L, Bekhuis T. De-duplication of database search results for systematic reviews in EndNote. J Med Libr Assoc. 2016;104(3):240-3.

22. Maslach C, Jackson SE, Leiter MP. Maslach burnout inventory manual, vol. iv. 3rd ed. Palo Alto: Consulting Psychologists Press; 1996. p. 52.

23. Assarroudi A, Heshmati Nabavi F, Armat MR, Ebadi A, Vaismoradi M. Directed qualitative content analysis: the description and elaboration of its underpinning methods and data analysis process. J Res Nurs. 2018;23(1):42-55.

24. Berta W, Laporte A, Perreira T, Ginsburg L, Dass AR, Deber R, et al. Relationships between work outcomes, work attitudes and work environments of health support workers in Ontario long-term care and home and community care settings. Hum Resour Health. 2018;16(1):15.

25. Adwan JZ. Pediatric nurses' grief experience, burnout and job satisfaction. J Pediatr Nurs. 2014;29(4):329-36

26. Akman O, Ozturk C, Bektas M, Ayar D, Armstrong MA. Job satisfaction and burnout among paediatric nurses. J Nurs Manag. 2016;24(7):923-33.

27. Alves DF, Guirardello EB. Safety climate, emotional exhaustion and job satisfaction among Brazilian paediatric professional nurses. Int Nurs Rev. 2016;63(3):328-35.

28. Amin AA, Vankar JR, Nimbalkar SM, Phatak AG. Perceived stress and professional quality of life in neonatal intensive care unit nurses in Gujarat. Indian J Pediatr. 2015;82(11):1001-5.

29. Aytekin A, Yilmaz F, Kuguoglu S. Burnout levels in neonatal intensive care nurses and its effects on their quality of life. Aust J Adv Nurs. 2013;31(2):39-47.

30. Barr P. Personality traits, state positive and negative affect, and professional quality of life in neonatal nurses. J Obst Gynecol Neonatal Nurs. 2018;22:22.

31. Barr P. The five-factor model of personality, work stress and professional quality of life in neonatal intensive care unit nurses. J Adv Nurs. 2018;74(6):1349-58.

32. Berger J, Polivka B, Smoot EA, Owens H. Compassion fatigue in pediatric nurses. J Pediatr Nurs. 2015;30(6):e11-7.

33. Bilal A, Ahmed HM. Organizational structure as a determinant of job burnout. Workplace Health Saf. 2017;65(3):118-28.

34. Bourbonnais R, Comeau M, Vezina M, Dion G. Job strain, psychological distress, and burnout in nurses. Am J Ind Med. 1998;34(1):20-8.

35. Branch C, Klinkenberg D. Compassion fatigue among pediatric healthcare providers. MCN Am J Matern Child Nurs. 2015;40(3):160-6 quiz E13-4.

36. Bursch B, Emerson ND, Arevian AC, Aralis H, Galuska L, Bushman J, et al. Feasibility of online mental wellness self-assessment and feedback for pediatric and neonatal critical care nurses. J Pediatr Nurs. 2018;43:62-8.

37. Czaja AS, Moss M, Mealer M. Symptoms of posttraumatic stress disorder among pediatric acute care nurses. J Pediatr Nurs. 2012;27(4):357-65.

38. Davis S, Lind BK, Sorensen C. A comparison of burnout among oncology nurses working in adult and pediatric inpatient and outpatient settings. Oncol Nurs Forum. 2013;40(4):E303-11.

39. Dos Santos Alves DF, da Silva D, de Brito Guirardello E. Nursing practice environment, job outcomes and safety climate: a structural equation modelling analysis. J Nurs Manag. 2017;25(1):46-55.

40. Downey $V$, Bengiamin $M$, Heuer $L$, Juhl N. Dying babies and associated stress in NICU nurses. Neonatal Netw. 1995;14(1):41-6.

41. Duxbury ML, Armstrong GD, Drew DJ, Henly SJ. Head nurse leadership style with staff nurse burnout and job satisfaction in neonatal intensive care units. Nurs Res. 1984;33(2):97-101.

42. Edmonds C, Lockwood GM, Bezjak A, Nyhof-Young J. Alleviating emotional exhaustion in oncology nurses: an evaluation of Wellspring's "Care for the Professional Caregiver Program". J Cancer Educ. 2012;27(1):27-36.

43. Estabrooks CA, Squires JE, Hutchinson AM, Scott S, Cummings GG, Kang SH, et al. Assessment of variation in the Alberta context tool: the contribution of unit level contextual factors and specialty in Canadian pediatric acute care settings. BMC Health Serv Res. 2011;11(1):251.

44. Faller MS, Gates MG, Georges JM, Connelly CD. Work-related burnout, job satisfaction, intent to leave, and nurse-assessed quality of care among travel nurses. J Nurs Adm. 2011;41(2):71-7.

45. Favrod C, Jan du Chene L, Martin Soelch C, Garthus-Niegel S, Tolsa JF, Legault $F$, et al. Mental health symptoms and work-related stressors in hospital midwives and NICU nurses: a mixed methods study. Front Psychiatry Front Res Found. 2018;9:364.
46. Gallagher R, Gormley DK. Perceptions of stress, burnout, and support systems in pediatric bone marrow transplantation nursing. Clin J Oncol Nurs. 2009;13(6):681-5

47. Gauthier T, Meyer RML, Grefe D, Gold Jl. An on-the-job mindfulness-based intervention for pediatric ICU nurses: a pilot. J Pediatr Nurs. 2015;30(2):402-9.

48. Gunusen NP, Wilson M, Aksoy B. Secondary traumatic stress and burnout among Muslim nurses caring for chronically ill children in a Turkish hospital. J Transcult Nurs. 2018;29(2):146-54.

49. Habadi Al, Alfaer SS, Shilli RH, Habadi MI, Suliman SM, Al-Aslany SJ, et al. The prevalence of burnout syndrome among nursing staff working at king Abdulaziz University hospital, Jeddah, Saudi Arabia, 2017. Divers Equality Health Care. 2018;15(3):122.

50. Hallberg IR. Systematic clinical supervision in a child psychiatric ward: satisfaction with nursing care, tedium, burnout, and the nurses' own report on the effects of it. Arch Psychiatr Nurs. 1994;8(1):44-52.

51. Holden RJ, Scanlon MC, Patel NR, Kaushal R, Escoto KH, Brown RL, et al. A human factors framework and study of the effect of nursing workload on patient safety and employee quality of working life. BMJ Qual Saf. 2011;20(1):15-24.

52. Hsu HY, Chen SH, Yu HY, Lou JH. Job stress, achievement motivation and occupational burnout among male nurses. J Adv Nurs. 2010;66(7):1592-601.

53. Hylton Rushton C, Batcheller J, Schroeder K, Donohue P. Burnout and resilience among nurses practicing in high-intensity settings. Am J Crit Care. 2015;24(5):412-21.

54. Jacobs LM, Nawaz MK, Hood JL, Bae S. Burnout among workers in a pediatric health care system. Workplace Health Saf. 2012;60(8):335-44.

55. Kase SM, Waldman ED, Weintraub AS. A cross-sectional pilot study of compassion fatigue, burnout, and compassion satisfaction in pediatric palliative care providers in the United States. Palliat Supportive Care. 2018;55(2):1-7.

56. Klein SD, Bucher HU, Hendriks MJ, Baumann-Holzle R, Streuli JC, Berger TM, et al. Sources of distress for physicians and nurses working in Swiss neonatal intensive care units. Swiss Med Wkly. 2017;147:w14477.

57. Koivula M, Paunonen M, Laippala P. Burnout among nursing staff in two Finnish hospitals. J Nurs Manag. 2000;8(3):149-58.

58. Latimer M, Jackson PL, Eugene F, MacLeod E, Hatfield T, Vachon-Presseau E, et al. Empathy in paediatric intensive care nurses part 1: behavioural and psychological correlates. J Adv Nurs. 2017;73(11):2676-85.

59. Lewiston NJ, Conley J, Blessing-Moore J. Measurement of hypothetical burnout in cystic fibrosis caregivers. Acta Paediatr Scand. 1981;70(6):935-9.

60. Li A, Early SF, Mahrer NE, Klaristenfeld JL, Gold Jl. Group cohesion and organizational commitment: protective factors for nurse residents' job satisfaction, compassion fatigue, compassion satisfaction, and burnout. J Prof Nurs. 2014:30(1):89-99.

61. Liakopoulou M, Panaretaki I, Papadakis V, Katsika A, Sarafidou J, Laskari H, et al. Burnout, staff support, and coping in pediatric oncology. Support Care Cancer. 2008;16(2):143-50.

62. Lin F, St John W, McVeigh C. Burnout among hospital nurses in China. J Nurs Manag. 2009;17(3):294-301.

63. Lin TC, Lin HS, Cheng SF, Wu LM, Ou-Yang MC. Work stress, occupational burnout and depression levels: a clinical study of paediatric intensive care unit nurses in Taiwan. J Clin Nurs. 2016;25(7-8):1120-30.

64. Liu X, Zheng J, Liu K, Baggs JG, Liu J, Wu Y, et al. Hospital nursing organizational factors, nursing care left undone, and nurse burnout as predictors of patient safety: a structural equation modeling analysis. Int J Nurs Stud. 2018;86:82-9.

65. Maytum JC, Heiman MB, Garwick AW. Compassion fatigue and burnout in nurses who work with children with chronic conditions and their families. J Pediatr Health Care. 2004;18(4):171-9.

66. Meadors P, Lamson A, Swanson M, White M, Sira N. Secondary traumatization in pediatric healthcare providers: compassion fatigue, burnout, and secondary traumatic stress. Omega. 2009;60(2):103.

67. Messmer PR, Bragg J, Williams PD. Support programs for new graduates in pediatric nursing. J Contin Educ Nurs. 2011;42(4):182-92.

68. Moody K, Kramer D, Santizo RO, Magro L, Wyshogrod D, Ambrosio J, et al. Helping the helpers: mindfulness training for burnout in pediatric oncology--a pilot program. J Pediatr Oncol Nurs. 2013;30(5):275-84.

69. Morelius E, Gustafsson PA, Ekberg K, Nelson N. Neonatal intensive care and child psychiatry inpatient care: do different working conditions influence stress levels? Nurs Res Pract. 2013;2013:761213.

70. Morrison Wylde C, Mahrer NE, Meyer RML, Gold Jl. Mindfulness for novice pediatric nurses: smartphone application versus traditional intervention. J Pediatr Nurs. 2017;36:205-12. 
71. Moussa I, Mahmood E. The relationship between nurses' burnout and mothers' satisfaction with pediatric nursing care. Int J Curr Res. 2013;5(7):1902-7.

72. Mudallal RH, Othman WM, Al Hassan NF. Nurses' burnout: the influence of leader empowering behaviors, work conditions, and demographic traits. Inquiry. 2017:54:46958017724944.

73. Murphy-Oikonen J, Brownlee K, Montelpare W, Gerlach K. The experiences of NICU nurses in caring for infants with neonatal abstinence syndrome. Neonatal Netw. 2010;29(5):307-13.

74. Neumann JL, Mau LW, Virani S, Denzen EM, Boyle DA, Boyle NJ, et al. Burnout, moral distress, work-life balance, and career satisfaction among hematopoietic cell transplantation professionals. Biol Blood Marrow Transplant. 2018;24(4):849-60.

75. Nguyen HTT, Kitaoka K, Sukigara M, Thai AL. Burnout study of clinical nurses in Vietnam: development of job burnout model based on Leiter and Maslach's theory. Asian Nurs Res. 2018;12(1):42-9.

76. Oehler JM, Davidson MG. Job stress and burnout in acute and nonacute pediatric nurses. Am J Crit Care. 1992;1(2):81-90.

77. Oehler JM, Davidson MG, Starr LE, Lee DA. Burnout, job stress, anxiety, and perceived social support in neonatal nurses. Heart Lung. 1991;20(5 Pt 1):500-5.

78. Ohue T, Moriyama M, Nakaya T. Examination of a cognitive model of stress, burnout, and intention to resign for Japanese nurses. Jpn J Nurs Sci. 2011;8(1):76-86.

79. Pagel I, Wittmann ME. Relationship of burnout to personal and job-related variables in acute-care pediatric settings. Issues Compr Pediatr Nurs. 1986; 9(2):131-43

80. Profit J, Sharek PJ, Amspoker AB, Kowalkowski MA, Nisbet CC, Thomas EJ, et al. Burnout in the NICU setting and its relation to safety culture. BMJ Qual Saf. 2014;23(10):806-13.

81. Richter LM, Rochat TJ, Hsiao C, Zuma TH. Evaluation of a brief intervention to improve the nursing care of young children in a high HIV and AIDS setting. Nurs Res Pract. 2012;2012:647182.

82. Robins PMP, Meltzer LP, Zelikovsky NP. The experience of secondary traumatic stress upon care providers working within a children's hospital. J Pediatr Nurs. 2009;24(4):270.

83. Rochefort CM, Clarke SP. Nurses' work environments, care rationing, job outcomes, and quality of care on neonatal units. J Adv Nurs. 2010;66(10):2213-24.

84. Rodrigues NP, Cohen LL, McQuarrie SC, Reed-Knight B. Burnout in nurses working with youth with chronic pain: a pilot intervention. J Pediatr Psychol. 2018:43(4):382-91.

85. Rodrigues NP, Cohen LL, Swartout KM, Trotochaud K, Murray E. Burnout in nurses working with youth with chronic pain: a mixed-methods analysis. J Pediatr Psychol. 2018;43(4):369-81.

86. Rodríguez-Rey R, Palacios A, Alonso-Tapia J, Pérez E, Álvarez E, Coca A, et al. Burnout and posttraumatic stress in paediatric critical care personnel: prediction from resilience and coping styles. Aust Crit Care. 2019;32(1):46-53.

87. Roney LN, Acri MC. The cost of caring: an exploration of compassion fatigue, compassion satisfaction, and job satisfaction in pediatric nurses. J Pediatr Nurs. 2018:40:74-80.

88. Sekol M, Kim S. Job satisfaction, burnout, and stress among pediatric nurses in various specialty units at an acute care hospital. J Nurs Educ Pract. 2014; $4(12): 115-24$.

89. Skorobogatova N, Zemaitiene N, Smigelskas K, Tameliene R. Professional burnout and concurrent health complaints in neonatal nursing. Open Med. 2017;12:328-34.

90. Soroush F, Zargham-Boroujeni A, Namnabati M. The relationship between nurses' clinical competence and burnout in neonatal intensive care units. Iran J Nurs Midwifery Res. 2016;21(4):424-9.

91. Squires JE, Estabrooks CA, Scott SD, Cummings GG, Hayduk L, Kang SH, et al. The influence of organizational context on the use of research by nurses in Canadian pediatric hospitals. BMC Health Serv Res. 2013;13(1):351.

92. Stimpfel AW, Lake ET, Barton S, Gorman KC, Aiken LH. How differing shift lengths relate to quality outcomes in pediatrics. J Nurs Adm. 2013;43(2):95-100.

93. Sun JW, Lin PZ, Zhang HH, Li JH, Cao FL. A non-linear relationship between the cumulative exposure to occupational stressors and nurses' burnout and the potentially emotion regulation factors. J Ment Health. 2017;27(5):1-7.

94. Sun WY, Ling GP, Chen P, Shan L. Burnout among nurses in the People's Republic of China. Int J Occup Environ Health. 1996;2(4):274-9.

95. Tawfik DS, Phibbs CS, Sexton JB, Kan P, Sharek PJ, Nisbet CC, et al. Factors associated with provider burnout in the NICU. Pediatrics. 2017;139(5):05.

96. Tawfik DS, Sexton JB, Kan P, Sharek PJ, Nisbet CC, Rigdon J, et al. Burnout in the neonatal intensive care unit and its relation to healthcare-associated infections. J Perinatol. 2017;37(3):315-20.
97. Vicentic S, Sapic R, Damjanovic A, Vekic B, Loncar Z, Dimitrijevic I, et al. Burnout of formal caregivers of children with cerebral palsy. Isr J Psychiatry Relat Sci. 2016;53(2):10-5.

98. Paula Vega V, Rina González R, Natalie Santibáñez G, Camila Ferrada M, Javiera Spicto O, Antonia Sateler $V$, et al. Supporting in grief and burnout of the nursing team from pediatric units in Chilean hospitals. Revista da Escola de Enfermagem da USP. 2017;51:1-6.

99. Watson P, Feld A. Factors in stress and burnout among paediatric nurses in a general hospital. Nurs Prax N Z. 1996;11(3):38-46.

100. Yao Y, Zhao S, Gao X, An Z, Wang S, Li H, et al. General self-efficacy modifies the effect of stress on burnout in nurses with different personality types. BMC Health Serv Res. 2018;18(1):667.

101. Zanatta AB, Lucca SR. Prevalence of burnout syndrome in health professionals of an onco-hematological pediatric hospital. Revista Da Escola de Enfermagem Da Usp. 2015;49(2):253-60.

102. Jourdain G, Chenevert D. Job demands-resources, burnout and intention to leave the nursing profession: a questionnaire survey. Int J Nurs Stud. 2010; 47(6):709-22.

103. Perreira TA, Berta W, Laporte A, Ginsburg L, Deber R, Elliott G, et al. Shining a light: examining similarities and differences in the work psychology of health support workers employed in long-term care and home and community care settings. J Appl Gerontol. 2017;38(11):0733464817737622.

104. Pinder CC. Work motivation in organizational behavior: psychology press; 2014

105. Cartwright S, Cooper CL. The Oxford handbook of organizational well-being. Oxford. New York: Oxford University Press; 2009. Available from: http:// myaccess.library.utoronto.ca/login?url=https://www.oxfordhandbooks.com/ view/10.1093/oxfordhb/9780199211913.001.0001/oxfordhb-9780199211913

106. Rotenstein LS, Torre M, Ramos MA, Rosales RC, Guille C, Sen S, et al. Prevalence of burnout among physicians: a systematic review. JAMA. 2018; 320(11):1131-50.

107. Chen SM, McMurray A. "Burnout" in intensive care nurses. J Nurs Res. 2001; 9(5):152-64

108. Poncet MC, Toullic P, Papazian L, Kentish-Barnes N, Timsit JF, Pochard F, et al. Burnout syndrome in critical care nursing staff. Am J Respir Crit Care Med. 2007;175(7):698-704.

109. Myhren H, Ekeberg O, Stokland O. Job satisfaction and burnout among intensive care unit nurses and physicians. Crit Care Res Pract. 2013;2013: 786176.

110. Adriaenssens J, De Gucht V, Maes S. Determinants and prevalence of burnout in emergency nurses: a systematic review of 25 years of research. Int J Nurs Stud. 2015;52(2):649-61.

111. Brown PA, Slater M, Lofters A. Personality and burnout among primary care physicians: an international study. Psychol Res Behav Manag. 2019;12:169-77.

112. McManus IC, Keeling A, Paice E. Stress, burnout and doctors' attitudes to work are determined by personality and learning style: a twelve year longitudinal study of UK medical graduates. BMC Med. 2004;2:29.

113. De la Fuente-Solana El, Gomez-Urquiza JL, Canadas GR, Albendin-Garcia L, Ortega-Campos E, Cañadas-De la Fuente GA. Burnout and its relationship with personality factors in oncology nurses. Eur J Oncol Nurs. 2017;30:91-6.

114. Shanafelt TD, Boone S, Tan L, Dyrbye LN, Sotile W, Satele D, et al. Burnout and satisfaction with work-life balance among US physicians relative to the general US Population. JAMA Intern Med. 2012;172(18):1377-85.

115. Moloney W, Boxall P, Parsons M, Cheung G. Factors predicting registered nurses' intentions to leave their organization and profession: a job demands-resources framework. J Adv Nurs. 2018;74(4):864-75.

116. Dall Ora C, Griffiths P, Ball J, Simon M, Aiken LH. Association of $12 \mathrm{~h}$ shifts and nurses' job satisfaction, burnout and intention to leave: findings from a crosssectional study of 12 European countries. BMJ Open. 2015;5(9):e008331.

117. Galvan ME, Vassallo JC, Rodriguez SP, Otero P, Montonati MM, Cardigni G, et al. Professional burnout in pediatric intensive care units in Argentina. Arch Argent Pediatr. 2012;110(6):466-73.

118. Garcia-Izquierdo M, Rios-Risquez MI. The relationship between psychosocial job stress and burnout in emergency departments: an exploratory study. Nurs Outlook. 2012;60(5):322-9.

119. Spence Laschinger HK, Grau AL, Finegan J, Wilk P. Predictors of new graduate nurses' workplace well-being: testing the job demands-resources model. Health Care Manag Rev. 2012;37(2):175-86.

120. Lindborg C, Davidhizar R. Is there a difference in nurse burnout on the day or night shift? Health Care Superv. 1993;11(3):47-52.

121. Shanafelt TD, Dyrbye LN, West CP. Addressing physician burnout: the way forward. JAMA. 2017;317(9):901-2. 
122. Shanafelt T, Trockel M, Ripp J, Murphy ML, Sandborg C, Bohman B. Building a program on well-being: key design considerations to meet the unique needs of each organization. Acad Med. 2018;21:21.

123. Hunsaker S, Chen HC, Maughan D, Heaston S. Factors that influence the development of compassion fatigue, burnout, and compassion satisfaction in emergency department nurses. J Nurs Scholarsh. 2015;47(2):186-94.

124. Cropanzano R, Mitchell M. Social exchange theory: an interdisciplinary review. J Manag. 2005;31(6):874-900.

125. Leiter MP, Maslach C. The impact of interpersonal environment on burnout and organizational commitment. J Organ Behav (1986-1998). 1988:9(4):297.

126. Teixeira C, Ribeiro O, Fonseca AM, Carvalho AS. Burnout in intensive care units - a consideration of the possible prevalence and frequency of new risk factors: a descriptive correlational multicentre study. BMC Anesthesiol. 2013;13(1):38.

127. Merecz D, Drabek M, Moscicka A. Aggression at the workplace-psychological consequences of abusive encounter with coworkers and clients. Int J Occup Med Environ Health. 2009;22(3):243-60.

128. Chang Y, Chan HJ. Optimism and proactive coping in relation to burnout among nurses. J Nurs Manag. 2015;23(3):401-8.

129. Ding $Y$, Yang $Y$, Yang $X$, Zhang $T$, Qiu $X$, He $X$, et al. The mediating role of coping style in the relationship between psychological capital and burnout among Chinese nurses. PLoS One. 2015:10(4):e0122128.

130. Laschinger HK, Grau AL. The influence of personal dispositional factors and organizational resources on workplace violence, burnout, and health outcomes in new graduate nurses: a cross-sectional study. Int J Nurs Stud. 2012:49(3):282-91.

131. Bakker AB, Le Blanc PM, Schaufeli WB. Burnout contagion among intensive care nurses. J Adv Nurs. 2005;51(3):276-87.

132. Aiken LH, Clarke SP, Sloane DM, Sochalski J, Silber JH. Hospital nurse staffing and patient mortality, nurse burnout, and job dissatisfaction. JAMA. 2002; 288(16):1987-93.

133. McHugh MD, Kutney-Lee A, Cimiotti JP, Sloane DM, Aiken LH. Nurses' widespread job dissatisfaction, burnout, and frustration with health benefits signal problems for patient care. Health Aff. 2011;30(2):202-10.

134. Cameron SJ, Horsburgh ME, Armstrong-Stassen M. Job satisfaction, propensity to leave and burnout in RNs and RNAs: a multivariate perspective. Can J Nurs Adm. 1994;7(3):43-64.

135. Leiter MP, Maslach C. Nurse turnover: the mediating role of burnout. J Nurs Manag. 2009:17(3):331-9.

136. Willard-Grace R, Knox M, Huang B, Hammer H, Kivlahan C, Grumbach K. Burnout and health care workforce turnover. Ann Fam Med. 2019;17(1):36-41.

137. Colville GA, Smith JG, Brierley J, Citron K, Nguru NM, Shaunak PD, et al. Coping with staff burnout and work-related posttraumatic stress in intensive care. Pediatr Crit Care Med. 2017;18(7):e267-e73.

138. Creedy DK, Sidebotham M, Gamble J, Pallant J, Fenwick J. Prevalence of burnout, depression, anxiety and stress in Australian midwives: a crosssectional survey. BMC Pregnancy Childbirth. 2017;17(1):13.

139. Salvagioni DAJ, Melanda FN, Mesas AE, Gonzalez AD, Gabani FL, Andrade SM. Physical, psychological and occupational consequences of job burnout: a systematic review of prospective studies. PLoS One. 2017;12(10):e0185781.

140. Vasconcelos EM, Martino MMF, Franca SPS. Burnout and depressive symptoms in intensive care nurses: relationship analysis. Rev Bras Enferm. 2018;71(1):135-41.

141. Welp A, Meier LL, Manser T. Emotional exhaustion and workload predict clinician-rated and objective patient safety. Front Psychol. 2014;5:1573.

142. Bodenheimer T, Sinsky C. From triple to quadruple aim: care of the patient requires care of the provider. Ann Fam Med. 2014;12(6):573-6.

143. Palsson MB, Hallberg IR, Norberg A, Bjorvell H. Burnout, empathy and sense of coherence among Swedish district nurses before and after systematic clinical supervision. Scand J Caring Sci. 1996;10(1):19-26.

144. Barbosa P, Raymond G, Zlotnick C, Wilk J, Toomey R III, Mitchell J III. Mindfulness-based stress reduction training is associated with greater empathy and reduced anxiety for graduate healthcare students. Educ Health. 2013;26(1):9-14.

145. de Vibe M, Solhaug I, Tyssen R, Friborg O, Rosenvinge JH, Sørlie T, et al. Mindfulness training for stress management: a randomised controlled study of medical and psychology students. BMC Med Educ. 2013;13(1):107.

146. Garneau K, Hutchinson T, ZHAO Q, Dobkin P. Cultivating person centered medicine in future physicians. Eur J Pers Cent Healthc. 2013;1(2):468-77.

147. Goodman MJ, Schorling JB. A mindfulness course decreases burnout and improves well-being among healthcare providers. Int J Psychiatry Med. 2012;43(2):119-28.
148. Cohen M, Gagin R. Can skill-development training alleviate burnout in hospital social workers? Soc Work Health Care. 2005;40(4):83-97.

149. Kravits K, McAllister-Black R, Grant M, Kirk C. Self-care strategies for nurses: a psycho-educational intervention for stress reduction and the prevention of burnout. Appl Nurs Res. 2010;23(3):130-8.

150. Sallon S, Katz-Eisner D, Yaffe H, Bdolah-Abram T. Caring for the caregivers: results of an extended, five-component stress-reduction intervention for hospital staff. Behav Med. 2017:43(1):47-60.

151. Wlodarczyk $N$. The effect of a group music intervention for grief resolution on disenfranchised grief of hospice workers. Prog Palliat Care. 2013;21(2):97-106.

152. Adams RJ, Smart P, Huff AS. Shades of grey: guidelines for working with the grey literature in systematic reviews for management and organizational studies. Int J Manag Rev. 2017;19(4):432-54.

\section{Publisher's Note}

Springer Nature remains neutral with regard to jurisdictional claims in published maps and institutional affiliations.
Ready to submit your research? Choose BMC and benefit from:

- fast, convenient online submission

- thorough peer review by experienced researchers in your field

- rapid publication on acceptance

- support for research data, including large and complex data types

- gold Open Access which fosters wider collaboration and increased citations

- maximum visibility for your research: over $100 \mathrm{M}$ website views per year

At $\mathrm{BMC}$, research is always in progress.

Learn more biomedcentral.com/submissions 